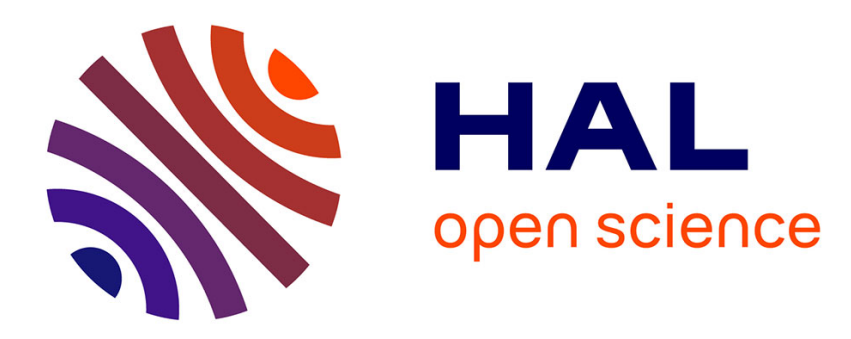

\title{
Motivic invariants of real polynomial functions and their Newton polyhedrons
}

Goulwen Fichou, Toshizumi Fukui

\section{To cite this version:}

Goulwen Fichou, Toshizumi Fukui. Motivic invariants of real polynomial functions and their Newton polyhedrons. Mathematical Proceedings of the Cambridge Philosophical Society, 2016, 160 (1), pp.141166. 10.1017/S030500411500064X . hal-00516080v2

\section{HAL Id: hal-00516080 \\ https://hal.science/hal-00516080v2}

Submitted on 14 Oct 2015

HAL is a multi-disciplinary open access archive for the deposit and dissemination of scientific research documents, whether they are published or not. The documents may come from teaching and research institutions in France or abroad, or from public or private research centers.
L'archive ouverte pluridisciplinaire HAL, est destinée au dépôt et à la diffusion de documents scientifiques de niveau recherche, publiés ou non, émanant des établissements d'enseignement et de recherche français ou étrangers, des laboratoires publics ou privés. 


\title{
MOTIVIC INVARIANTS OF REAL POLYNOMIAL FUNCTIONS AND THEIR NEWTON POLYHEDRONS
}

\author{
GOULWEN FICHOU AND TOSHIZUMI FUKUI
}

\begin{abstract}
We give an expression of the motivic zeta function for a real polynomial function in terms of the Newton polyhedron of the function. As a consequence, we show that the weights are determined by the motivic zeta function for convenient weighted homogeneous polynomials in three variables. We apply this result to the blow-Nash equivalence.
\end{abstract}

In Singularity Theory, one aim is to classify singular objects with respect to a given equivalence relation. We focus on the singularities of function germs, and more precisely on the case of weighted homogeneous polynomial functions; that is, polynomial functions that satisfy

$$
f\left(t^{w_{1}} x_{1}, \ldots, t^{w_{n}} x_{n}\right)=t^{d} f\left(x_{1}, \ldots, x_{n}\right) \quad \text { for all real numbers } t .
$$

We refer to $\left(w_{1}, \ldots, w_{n}\right) \in \mathbb{N}^{n}$ as weights and to $d \in \mathbb{N}$ as the weighted degree of $f$ with respect to $\left(w_{1}, \ldots, w_{n}\right)$. We tackle the question of the invariance of the weights under a given equivalence relation for germs of such functions at the origin.

Concerning complex analytic function germs, the first result in this direction is due to K. Saito ([19, Lemma 4.3]). He proved that, among the weighted homogeneous polynomials with isolated singularities, the weights are local analytic invariants of the pair $\left(\mathbb{C}^{n}, f^{-1}(0)\right)$ at the origin, for a weighted homogeneous polynomial $f$. Concerning the topological equivalence, E. Yoshinaga and M. Suzuki [20] in 1979 (and later T. Nishimura [17] in 1986) proved the topological invariance of the weights in the two variable case with isolated singularities, whereas O. Saeki [18] in 1988 proved that the weights of a weighted homogeneous polynomial $f$ in $\mathbb{C}^{3}$ with an isolated singularity are local topological invariants of the pair $\left(\mathbb{C}^{3}, f^{-1}(0)\right)$ at the origin.

In this paper, we are concerned with the real counterpart of this question, considering equivalence relations on real analytic function germs. Since the topological equivalence is too weak in the real setting, the most relevant equivalence relation to consider is the blow-analytic equivalence introduced by T.-C. Kuo (cf. [15], and also $[10,11]$ for surveys). Real analytic function germs $f, g:\left(\mathbb{R}^{n}, 0\right) \longrightarrow(\mathbb{R}, 0)$ are said to be blow-analytically equivalent in the sense of [15] if there exist real modifications $\beta_{f}: M_{f} \longrightarrow \mathbb{R}^{n}$ and $\beta_{g}: M_{g} \longrightarrow \mathbb{R}^{n}$ and an analytic isomorphism $\Phi:\left(M_{f}, \beta_{f}^{-1}(0)\right) \longrightarrow\left(M_{g}, \beta_{g}^{-1}(0)\right)$ which induces a homeomorphism $\phi:\left(\mathbb{R}^{n}, 0\right) \longrightarrow$ $\left(\mathbb{R}^{n}, 0\right)$ such that $f=g \circ \phi$.

Date: October 14, 2015.

2000 Mathematics Subject Classification. 14P20 (14B05 14P25 32S15).

Key words and phrases. Zeta functions, virtual Betti numbers, blow-Nash equivalence.

The authors have been supported by Saitama University and the ANR project ANR-08-JCJC0118-01. 
For polynomial functions, or more generally Nash functions (i. e., real analytic functions with semi-algebraic graphs), a natural counterpart exists, called blowNash equivalence, that takes into account the algebraic nature of Nash functions. This equivalence relation has been proved to have nice properties (cf. [10, 7, 8]).

The question of the invariance of the weights for weighted homogeneous polynomial functions under blow-analytic equivalence already appeared as a conjecture in [9] and as a question in [13]. A positive answer has been given by O. M. Abderrahmane [1] in the two variable case, using two invariants of blow-analytic equivalence: Fukui invariants [9] and zeta functions [13] constructed by S. Koike and A. Parusiński using motivic integration [5] with the Euler characteristic of the homology of locally finite chains with closed supports as a measure.

In the case of blow-Nash equivalence, the forthcoming Theorem 4.9 states that convenient three variable weighted homogeneous polynomial functions that are blowNash equivalent must have the same weights. To prove this, we investigate the zeta function introduced in [7] as an invariant of the blow-Nash equivalence, using as a measure the virtual Poincaré polynomial [16]. This polynomial is an additive and multiplicative invariant for real algebraic sets, whose degree is equal to the dimension of the variety.

The main point in the proof of Theorem 4.9 is to estimate the degrees of the coefficients of the zeta functions, which are defined using the virtual Poincaré polynomial, in terms of the Newton polyhedron of a given polynomial function. Zeta functions in motivic integration have already been computed in terms of the Newton polyhedron [3, 4, 12], and our Theorem 2.6 is a version that focusses on bounds on the degrees. The main result in this paper, Theorem 3.10, gives a bound for the degree of the coefficients of the zeta function, which leads to the notion of leading exponent in section 3.2. In the case of convenient weighted homogeneous polynomial functions, this leading exponent gives one more information on the weights. This information will be sufficient to conclude Theorem 4.9.

Notation: Throughout the paper, $\mathbb{R}$ denotes the set of real numbers, $\mathbb{N}$ denotes the set of natural numbers with 0 and we set

$$
\mathbb{R}_{+}=\{x \in \mathbb{R}: x \geq 0\}, \quad \mathbb{R}_{+}^{*}=\{x \in \mathbb{R}: x>0\}, \quad \mathbb{N}^{*}=\mathbb{N} \backslash\{0\}
$$

Acknowledgements: The authors would like to thank the anonymous referee for their useful comments in order to improve the paper.

\section{Motivic MeAsure For ARC SPACE}

In this section we recall briefly how we can measure certain subsets of arc spaces in the context of real geometry, using the theory of motivic integration as developed by J. Denef and F. Loeser [5]. The real theory has already been developed in [13, 7].

The measure takes its value in the Grothendieck ring $K_{0}\left(\operatorname{Var}_{\mathbb{R}}\right)$ of real algebraic varieties [16]. It is defined as the free abelian group $K_{0}\left(\operatorname{Var}_{\mathbb{R}}\right)$ generated by isomorphism classes $[X]$ of real algebraic varieties modulo the subgroup generated by the relation $[X]=[Y]+[X \backslash Y]$ for $Y \subset X$ a closed subvariety of $X$. The multiplicative structure comes from the Cartesian product of varieties. 
1.1. Motivic zeta functions. Let $M$ be a nonsingular real algebraic variety and $S$ a subset of $M$. Consider the space of formal arcs with origin in $S$

$$
\mathcal{L}(M, S):=\{\alpha:(\mathbb{R}, 0) \rightarrow(M, S): \text { formal }\} .
$$

We write $\mathcal{L}(M, x)$ when $S=\{x\}$ is reduced to a point, and for $m \in \mathbb{N}$ denote by $L_{m}(M, x)$ the set of $m$-jets of elements of $\mathcal{L}(M, x)$.

Let $p_{m}: \mathcal{L}\left(\mathbb{R}^{n}, 0\right) \rightarrow L_{m}\left(\mathbb{R}^{n}, 0\right)$ denote the map defined by taking $m$-jets. For a subset $\mathcal{A}$ of $\mathcal{L}\left(\mathbb{R}^{n}, 0\right)$ which is constructible in the sense of [6], then, for each $m \in \mathbb{N}, p_{m}(\mathcal{A})$ is Zariski constructible and therefore admits a measure in $K_{0}\left(\operatorname{Var}_{\mathbb{R}}\right)$. Moreover the limit

$$
\lim _{m \rightarrow \infty} \frac{\left[p_{m}(\mathcal{A})\right]}{\mathbb{L}^{m n}}
$$

has a meaning in the localised Grothendieck ring $K_{0}\left(\operatorname{Var}_{\mathbb{R}}\right)\left[\left[\mathbb{L}^{-1}\right]\right]$, where $\left[p_{m}(\mathcal{A})\right]$ is the measure of $p_{m}(\mathcal{A})$ in $K_{0}\left(\operatorname{Var}_{\mathbb{R}}\right)$ and $\mathbb{L}$ the measure of the real affine line. We define the measure of $\mathcal{A}$ to be this limit.

A typical example of such a constructible subset of $\mathcal{L}\left(\mathbb{R}^{n}, 0\right)$ is given by the preimage under a truncation map $p_{m}$ of a Zariski constructible subset of $L_{m}(M, x)$ (cf. [5]). The subsets of the arc space we consider in this paper are of this type.

The particular case of spaces of arcs defined by arcs with a specified order will play a crucial role in this paper.

Example 1.1. For $a=\left(a_{1} \ldots, a_{n}\right) \in \mathbb{N}^{n}$, we consider the set $\mathcal{L}_{a} \subset \mathcal{L}\left(\mathbb{R}^{n}, 0\right)$ of $\operatorname{arcs} \alpha=\left(\alpha_{1}, \ldots, \alpha_{n}\right)$ in $\mathbb{R}^{n}$ whose $i$-th component $\alpha_{i}$ vanishes if $a_{i}=0$ or $\alpha_{i}(t)=$ $c t^{a_{i}}+\ldots$, where $c \neq 0$, is of order $a_{i}$ otherwise. Namely

$$
\mathcal{L}_{a}=\left\{\alpha \in \mathcal{L}\left(\mathbb{R}^{n}, 0\right): \text { ord } \alpha_{i}=a_{i} \text { if } i \in I(a), \alpha_{i}=0 \text { if } i \notin I(a)\right\}
$$

where $I(a)=\left\{i: a_{i}>0\right\}$. If $m$ is greater than the maximal value of $a_{i}$, for $i=1, \ldots, n$, then $p_{m}\left(\mathcal{L}_{a}\right)$ is isomorphic to $\mathbb{R}^{m|I(a)|-\sum_{i} a_{i}} \times\left(\mathbb{R}^{*}\right)^{|I(a)|}$ where $|I(a)|$ denotes the cardinal of $I(a)$. Then

$$
\left[p_{m}\left(\mathcal{L}_{a}\right)\right]=\mathbb{L}^{m|I(a)|-\sum_{i} a_{i}}(\mathbb{L}-1)^{|I(a)|}=(\mathbb{L}-1)^{|I(a)|} \mathbb{L}^{m|I(a)|-s(a)}
$$

where $s(a)=\sum_{i=1}^{n} a_{i}$, and therefore

$$
\left[\mathcal{L}_{a}\right]=\lim _{m \rightarrow \infty} \frac{\left[p_{m}\left(\mathcal{L}_{a}\right)\right]}{\mathbb{L}^{m n}}= \begin{cases}(\mathbb{L}-1)^{n} \mathbb{L}^{-s(a)} & \text { if }|I(a)|=n, \\ 0 & \text { if }|I(a)|<n .\end{cases}
$$

In other words, truncated arcs with some components equal to zero, can be seen as the image under truncation of arcs with higher orders. We will use this remark in order to compute in section 2.2 the arc spaces associated with a given real polynomial function germ.

Let $f:\left(\mathbb{R}^{n}, 0\right) \rightarrow(\mathbb{R}, 0)$ be a polynomial function germ, and for $k \in \mathbb{N}^{*}$, we define the arc space $\mathcal{A}_{k}(f) \subset \mathcal{L}\left(\mathbb{R}^{n}, 0\right)$ by

$$
\mathcal{A}_{k}(f)=\left\{\alpha \in \mathcal{L}\left(\mathbb{R}^{n}, 0\right): f \circ \alpha(t)=c t^{k}+\cdots, c \neq 0\right\} .
$$

Similarly, we define arc spaces with $\operatorname{sign} \mathcal{A}_{k}^{+}(f) \subset \mathcal{A}_{k}(f)$ and $\mathcal{A}_{k}^{-}(f) \subset \mathcal{A}_{k}(f)$, by

$$
\mathcal{A}_{k}^{+}(f)=\left\{\alpha \in \mathcal{L}\left(\mathbb{R}^{n}, 0\right): f \circ \alpha(t)=c t^{k}+\cdots, c=1\right\}
$$

and

$$
\mathcal{A}_{k}^{-}(f)=\left\{\alpha \in \mathcal{L}\left(\mathbb{R}^{n}, 0\right): f \circ \alpha(t)=c t^{k}+\cdots, c=-1\right\} .
$$


As the treatment of the spaces with sign $\mathcal{A}_{k}^{+}(f)$ and $\mathcal{A}_{k}^{-}(f)$ is similar, we denote in the following by $\mathcal{A}_{k}^{ \pm}(f)$ one of these two spaces. Since the $k$-jet of $\alpha \in \mathcal{L}\left(\mathbb{R}^{n}, 0\right)$ determines the $k$-jet of $f \circ \alpha$, we obtain an expression for the measure of $\mathcal{A}_{k}(f)$ and of $\mathcal{A}_{k}^{ \pm}(f)$ in terms of the space of $m$-jets of arcs with $m \geq k$. More precisely

$$
\left[\mathcal{A}_{k}(f)\right]=\frac{\left[p_{m}\left(\mathcal{A}_{k}(f)\right)\right]}{\mathbb{L}^{m n}} \text { and }\left[\mathcal{A}_{k}^{ \pm}(f)\right]=\frac{\left[p_{m}\left(\mathcal{A}_{k}^{ \pm}(f)\right)\right]}{\mathbb{L}^{m n}}
$$

for $m \geq k$. The associated zeta function and zeta functions with sign are the formal power series with coefficients in $K_{0}\left(\operatorname{Var}_{\mathbb{R}}\right)\left[\left[\mathbb{L}^{-1}\right]\right]$ defined by

$$
Z(f)=\sum_{k=1}^{\infty}\left[\mathcal{A}_{k}(f)\right] t^{k} \text { and } Z^{ \pm}(f)=\sum_{k=1}^{\infty}\left[\mathcal{A}_{k}^{ \pm}(f)\right] t^{k}
$$

Example 1.2. Consider the one variable polynomial function defined by $f(x)=x^{d}$ with $d \in \mathbb{N}^{*}$. Then we have

$$
\left[\mathcal{A}_{k}(f)\right]= \begin{cases}(\mathbb{L}-1) \mathbb{L}^{-l} & \text { if } k=l d, l \in \mathbb{N}, \\ 0 & \text { if } d \nmid k .\end{cases}
$$

As a consequence, the zeta function of $f$ is equal to

$$
Z(f)=\mathbb{L}^{-1} \frac{(\mathbb{L}-1) t^{d}}{1-\mathbb{L}^{-1} t^{d}}
$$

1.2. The virtual Poincaré polynomial. For real algebraic varieties, the best additive invariant known is the virtual Poincaré polynomial [16]. It assigns a polynomial $\beta(X)$ with integer coefficients to a (real algebraic) Zariski constructible set $X$ in such a way that:

- $\beta(X)=\beta(Y)+\beta(X \backslash Y)$ for $Y \subset X$ a closed subvariety of $X$ (additivity),

- $\beta(X \times Y)=\beta(X) \beta(Y)$ (multiplicativity), and

- the coefficients coincide with the Betti numbers with $\mathbb{Z}_{2}$-coefficients for compact nonsingular real algebraic sets.

Proposition $1.3([16])$. Take $i \in \mathbb{N}$. The Betti number $\beta_{i}(\cdot)=\operatorname{dim} H_{i}\left(\cdot, \mathbb{Z}_{2}\right)$, considered on compact nonsingular real algebraic sets, admits a unique extension as an additive map $\beta_{i}$ to the category of Zariski constructible sets, with values in $\mathbb{Z}$. Namely

$$
\beta_{i}(X)=\beta_{i}(Y)+\beta_{i}(X \backslash Y)
$$

for $Y \subset X$ a closed subvariety of $X$.

Moreover the polynomial $\beta(\cdot)=\sum_{i \geq 0} \beta_{i}(\cdot) u^{i} \in \mathbb{Z}[u]$ is multiplicative

$$
\beta(X \times Y)=\beta(X) \beta(Y)
$$

for $X, Y$ Zariski constructible sets.

The invariant $\beta_{i}$ is called the $i$-th virtual Betti number, and the polynomial $\beta$ the virtual Poincaré polynomial. By evaluating of the virtual Poincaré polynomial at $u=-1$ one recovers the Euler characteristic for Borel-Moore homology, that is, the homology of locally finite chains with closed supports [16]. The following simple example illustrates the way to compute, in practice, the virtual Poincaré polynomial. 
Example 1.4. Let $\mathbb{P}^{n}$ denote the real projective space of dimension $n$, which is nonsingular and compact. Then $\beta\left(\mathbb{P}^{n}\right)=1+u+\cdots+u^{n}$, since

$$
\operatorname{dim} H_{i}\left(\mathbb{P}^{n}, \mathbb{Z}_{2}\right)= \begin{cases}1 & \text { for } i \in\{0, \ldots, n\}, \text { and } \\ 0 & \text { otherwise. }\end{cases}
$$

Now, compactify the affine line $\mathbb{A}_{\mathbb{R}}^{1}$ in $\mathbb{P}^{1}$ by adding one point $P$ at infinity. By additivity $\beta\left(\mathbb{A}_{\mathbb{R}}^{1}\right)=\beta\left(\mathbb{P}^{1}\right)-\beta(P)=u$, and so $\beta\left(\mathbb{A}_{\mathbb{R}}^{n}\right)=u^{n}$ by multiplicativity.

A crucial property of the virtual Poincaré polynomial is that the degree of $\beta(X)$ is equal to the dimension of the Zariski constructible set $X$. In particular, the virtual Poincaré polynomial of a constructible set $X$ cannot be equal to zero as soon as the set of real points of $X$ is not empty. We consider in this paper the virtual Poincaré polynomial of the (infinite dimensional) spaces of $\operatorname{arcs} \mathcal{A}_{k}(f)$ and $\mathcal{A}_{k}^{ \pm}(f)$ associated with a polynomial function $f$, for $k \in \mathbb{N}^{*}$. It is defined, e.g. in the case without sign and as explained in section 1.1, by the formula

$$
\beta\left(\mathcal{A}_{k}(f)\right)=\lim _{m \rightarrow \infty} \frac{\beta\left(p_{m}\left(\mathcal{A}_{k}\right)\right)}{u^{m n}} .
$$

We will mainly focus on the degree of $\beta\left(\mathcal{A}_{k}(f)\right)$ in section 3 , and we will also discuss its zeros in section 4 .

\section{Arc spaCes And NeWton polyhedrons}

In this section we are interested in expressing the measure of the arc spaces associated with a polynomial function germ in terms of its Newton polyhedron. Similar results have already been obtained in $[3,4,12]$. Here we focus mainly on a formula that will enable us to estimate efficiently the degree of the virtual Poincaré polynomial of the arc spaces in terms of the Newton polyhedron of the germ.

We start with introducing some standard notation for the Newton polyhedron associated with a polynomial.

2.1. The Newton polyhedron. Let $f: \mathbb{R}^{n} \rightarrow \mathbb{R}$ denote a polynomial function. Consider its Taylor expansion at the origin of $\mathbb{R}^{n}$

$$
f(x)=\sum_{\nu \in \mathbb{N}^{n}} c_{\nu} x^{\nu}
$$

where $x^{\nu}=\prod_{i=1}^{n} x_{i}^{\nu_{i}}$ with $\nu=\left(\nu_{1}, \ldots, \nu_{n}\right) \in \mathbb{N}^{n}$, and $c_{\nu} \in \mathbb{R}$. Let $\Gamma_{+}(f)$ denote the Newton polyhedron of $f$, defined as the convex hull of the set

$$
\cup_{\nu \in \mathbb{N}^{n}}\left(\nu+\mathbb{R}_{+}^{n}\right): c_{\nu} \neq 0,
$$

where $\mathbb{R}_{+}$stands for $[0,+\infty)$. The Newton boundary $\Gamma(f)$ of $f$ is the union of the compact faces of $\Gamma_{+}(f)$. We write $\gamma<\Gamma(f)$ to denote that the compact face $\gamma$ belongs to $\Gamma(f)$, and $\gamma<\sigma$ for the inclusion of two faces. For $a=\left(a_{1}, \ldots, a_{n}\right) \in \mathbb{R}_{+}^{n}$ and $\nu=\left(\nu_{1}, \ldots, \nu_{n}\right) \in \mathbb{R}^{n}$, we set $\langle a, \nu\rangle=\sum_{i=1}^{n} a_{i} \nu_{i}$ and define the multiplicity $m_{f}(a)$ of $f$ relative to $a$ by

$$
m_{f}(a)=\min \left\{\langle a, \nu\rangle: \nu \in \Gamma_{+}(f)\right\} .
$$

For $a \in \mathbb{R}_{+}^{n}$, we define the face $\gamma_{f}(a)$ of the Newton polyhedron of $f$ associated with $a$ by

$$
\gamma_{f}(a)=\left\{\nu \in \Gamma_{+}(f):\langle a, \nu\rangle=m_{f}(a)\right\}
$$


and for a subset $S$ of $\mathbb{N}^{n}$ we set

$$
f_{S}(x)=\sum_{\nu \in S} c_{\nu} x^{\nu}
$$

We define an equivalence relation on $\mathbb{R}_{+}^{n}$ by

$$
a \sim b \quad \gamma_{f}(a)=\gamma_{f}(b) .
$$

The partition of $\mathbb{R}_{+}^{n}$ given by the equivalence classes of this equivalence relation is the dual Newton diagram of $f$ and it is denoted by $\Gamma^{*}(f)$. This defines a cone subdivision of $\mathbb{R}_{+}^{n}$, which we identify with the dual Newton polyhedron throughout the paper. Note that the function $m_{f}$ is a piecewise linear function on $\mathbb{R}_{+}^{n}$, which is linear on any cone belonging to this subdivision. In particular, on the cone corresponding to a face $\gamma$ of $\Gamma_{+}(f)$, the function $m_{f}$ is given by the scalar product $\langle\cdot, \nu\rangle$ with any $\nu \in \gamma$. Let $\Lambda(f) \subset \mathbb{N}^{n}$ denote the set of primitive generators of the 1-cones of $\Gamma^{*}(f)$.

The polynomial $f$ is said to be convenient if the monomials $x_{i}^{p_{i}}$, for $i=1, \ldots, n$ and some $p_{i} \in \mathbb{N}^{*}$, appear in the expression of $f$ with non-zero coefficients.

We say that the polynomial function $f$ is non-degenerate if, for any compact face $\gamma$ of $\Gamma_{+}(f)$, all singular points of $f_{\gamma}$ are contained in the union of some coordinate hyperplanes. Namely $f$ is non-degenerate if

$$
\left(\frac{\partial f_{\gamma}}{\partial x_{1}}(c), \ldots, \frac{\partial f_{\gamma}}{\partial x_{n}}(c)\right) \neq(0, \ldots, 0)
$$

for all $c \in\left(\mathbb{R}^{*}\right)^{n}$ with $f_{\gamma}(c)=0$, where $\gamma$ is any compact face of $\Gamma_{+}(f)$.

Finally, for any compact face $\gamma$ of the Newton polyhedron of $f$, we define algebraic subsets $X_{\gamma}, X_{\gamma}^{+}$and $X_{\gamma}^{-}$of $\left(\mathbb{R}^{*}\right)^{n}$ by

$$
X_{\gamma}=\left\{c \in\left(\mathbb{R}^{*}\right)^{n}: f_{\gamma}(c)=0\right\}, \quad \text { and } \quad X_{\gamma}^{ \pm}=\left\{c \in\left(\mathbb{R}^{*}\right)^{n}: f_{\gamma}(c)= \pm 1\right\} .
$$

Remark 2.1. There exists an algebraic variety $\widehat{X}_{\gamma}$ in $\left(\mathbb{R}^{*}\right)^{\operatorname{dim} \gamma}$ so that

$$
X_{\gamma} \simeq\left(\mathbb{R}^{*}\right)^{n-\operatorname{dim} \gamma} \times \widehat{X}_{\gamma}
$$

(cf. [2], Vol. 2, Part II, Chapter 8 for example). Taking measures, we therefore have

$$
\left[X_{\gamma}\right]=(\mathbb{L}-1)^{n-\operatorname{dim} \gamma}\left[\widehat{X}_{\gamma}\right] .
$$

Example 2.2. Let $X$ denote the zero set of $f\left(x_{1}, x_{2}\right)=x_{1}^{2}+x_{2}^{3}$ in $\left(\mathbb{R}^{*}\right)^{2}$. We have $X \simeq \mathbb{R}^{*} \times \widehat{X}$ where $\widehat{X}=\left\{y_{2} \in \mathbb{R}^{*}: y_{2}+1=0\right\}$. This follows from the identity $f \circ \varphi\left(y_{1}, y_{2}\right)=y_{1}^{6} y_{2}^{2}\left(1+y_{2}\right)$, where $\varphi:\left(\mathbb{R}^{*}\right)^{2} \rightarrow\left(\mathbb{R}^{*}\right)^{2}$ is the algebraic isomorphism defined by $\left(y_{1}, y_{2}\right) \mapsto\left(x_{1}, x_{2}\right)=\left(y_{1}^{3} y_{2}, y_{1}^{2} y_{2}\right)$.

Remark 2.3. If $f$ is non-degenerate, then $X_{\gamma}$ (resp. $\widehat{X}_{\gamma}$ ) is a non-singular submanifold of $\left(\mathbb{R}^{*}\right)^{n}$ (resp. $\left(\mathbb{R}^{*}\right)^{\operatorname{dim} \gamma}$ ) of codimension 1 , whenever it is not empty. Since $f_{\gamma}$ is weighted homogeneous,

$$
\left\{\frac{\partial f_{\gamma}}{\partial x_{1}}=\cdots=\frac{\partial f_{\gamma}}{\partial x_{n}}=0\right\}=\left\{\frac{\partial f_{\gamma}}{\partial x_{1}}=\cdots=\frac{\partial f_{\gamma}}{\partial x_{n}}=f_{\gamma}=0\right\}
$$

and the non-degeneracy of $f$ also implies that the varieties $X_{\gamma}^{ \pm}$are nonsingular manifolds of codimension 1 , whenever they are not empty. Moreover, the differential of $f_{\gamma}$ is nonzero. Thus we have: 
- $X_{\gamma}^{+}$and $X_{\gamma}^{-}$cannot be empty simultaneously, and

- if $X_{\gamma}$ is not empty, then both $X_{\gamma}^{+}$and $X_{\gamma}^{-}$are not empty.

2.2. Motivic invariant of a polynomial function. We express the measure of the arc spaces associated with a polynomial function $f$ in terms of its Newton polyhedron. The set of integers $k \in \mathbb{N}^{*}$ for which the arc space $\mathcal{A}_{k}(f)$ is not empty has already been studied in the context of blow-analytic equivalence and is called the set of Fukui invariants $[1,13]$. It coincides with the set of exponents that appear in the zeta function of $f$ with non-zero coefficients. We denote by

$$
A(f)=\left\{k \in \mathbb{N}^{*}: \mathcal{A}_{k}(f) \neq \emptyset\right\}
$$

the set $A(f)$ of Fukui invariants of $f$ and by

$$
A^{ \pm}(f)=\left\{k \in \mathbb{N}^{*}: \mathcal{A}_{k}^{ \pm}(f) \neq \emptyset\right\} .
$$

the sets $A^{+}(f)$ and $A^{-}(f)$ of Fukui invariants with sign of $f$.

Next lemma (adapted to the real setting from [12], Lemma 2.2.1) computes the measure of the arc spaces associated with $f$ for arcs with a specified order $a \in \mathbb{N}^{n}$. As illustrated in Example 1.1, those arcs with order $a \in\left(\mathbb{N}^{*}\right)^{n}$ play the most important role when computing the measure.

Lemma 2.4. Assume that $f$ is a non-degenerate polynomial. Take $a \in\left(\mathbb{N}^{*}\right)^{n}$ and $k \in \mathbb{N}^{*}$. The measure of $\mathcal{L}_{a} \cap \mathcal{A}_{k}(f)$ is given by

$$
\left[\mathcal{L}_{a} \cap \mathcal{A}_{k}(f)\right]= \begin{cases}0 & \text { if } m_{f}(a)>k, \\ \left((\mathbb{L}-1)^{n}-\left[X_{\gamma(a)}\right]\right) \mathbb{L}^{-s(a)} & \text { if } m_{f}(a)=k, \\ (\mathbb{L}-1)\left[X_{\gamma(a)}\right] \mathbb{L}^{-s(a)-k+m_{f}(a)} & \text { if } m_{f}(a)<k .\end{cases}
$$

In the case with sign, the measure of $\mathcal{L}_{a} \cap \mathcal{A}_{k}^{ \pm}(f)$ is given by

$$
\left[\mathcal{L}_{a} \cap \mathcal{A}_{k}^{ \pm}(f)\right]= \begin{cases}0 & \text { if } m_{f}(a)>k, \\ {\left[X_{\gamma(a)}^{ \pm}\right] \mathbb{L}^{-s(a)}} & \text { if } m_{f}(a)=k, \\ {\left[X_{\gamma(a)}\right] \mathbb{L}^{-s(a)-k+m_{f}(a)}} & \text { if } m_{f}(a)<k .\end{cases}
$$

Proof. Since the proof of the case with sign is similar to the case without sign, we treat only the later. For an $\operatorname{arc} \alpha \in \mathcal{L}_{a}$ with order $a=\left(a_{1}, \ldots, a_{n}\right)$, we compute its composition with $f$ in terms of data coming from the Newton polyhedron of $f$. Define $\phi(t)=\left(\phi_{1}(t), \ldots, \phi_{n}(t)\right)$ by

$$
\alpha(t)=\left(t^{a_{1}} \phi_{1}(t), \ldots, t^{a_{n}} \phi_{n}(t)\right),
$$

so that $\phi_{i}(0) \neq 0$ for $i=1, \ldots, n$. Using the Taylor expansion for $f$, we obtain

$$
f(\alpha(t))=\sum_{\nu \in \mathbb{N}^{n}} c_{\nu} \prod_{i=1}^{n}\left(t^{a_{i}} \phi_{i}(t)\right)^{\nu_{i}}=\sum_{\nu \in \mathbb{N}^{n}} c_{\nu} \phi(t)^{\nu} t^{\langle a, \nu\rangle} .
$$

As the order of $f(\alpha(t))$ is greater than $m_{f}(a)$, we may write

$$
f(\alpha(t))=t^{m_{f}(a)}\left(f_{\gamma(a)}(\phi(t))+R(t)\right)
$$

where $R(t)$ is a power series, depending on $\phi$, with strictly positive order. In particular, if $k$ is strictly less than $m_{f}(a)$, then $\mathcal{L}_{a} \cap \mathcal{A}_{k}(f)$ is empty. If $k$ is equal to $m_{f}(a)$, then $\mathcal{L}_{a} \cap \mathcal{A}_{k}(f)$ is described by those arcs $\alpha$ with $f_{\gamma(a)}(\phi(0)) \neq 0$, meaning 
that $\phi(0) \in\left(\mathbb{R}^{*}\right)^{n} \backslash X_{\gamma(a)}$. To compute the measure of $\mathcal{L}_{a} \cap \mathcal{A}_{k}(f)$, note that the quotient

$$
\frac{\left[p_{m}\left(\mathcal{L}_{a} \cap \mathcal{A}_{k}(f)\right)\right]}{\mathbb{L}^{m n}}
$$

stabilizes for $m \geq k$, and for $m=k$ we have just seen that

$$
p_{k}\left(\mathcal{L}_{a} \cap \mathcal{A}_{k}(f)\right) \simeq\left(\left(\mathbb{R}^{*}\right)^{n} \backslash X_{\gamma(a)}\right) \times \mathbb{R}^{\sum_{i=1}^{n}\left(k-a_{i}\right)}
$$

so that

$$
\left[\mathcal{L}_{a} \cap \mathcal{A}_{k}(f)\right]=\frac{\left[\left(\left(\mathbb{R}^{*}\right)^{n} \backslash X_{\gamma(a)}\right) \times \mathbb{R}^{\sum_{i=1}^{n}\left(k-a_{i}\right)}\right]}{\mathbb{L}^{k n}}=\left((\mathbb{L}-1)^{n}-\left[X_{\gamma(a)}\right]\right) \mathbb{L}^{-s(a)} .
$$

We focus now on the case where $k$ is strictly bigger than $m_{f}(a)$. In this case, setting $F(t)=t^{-m_{f}(a)} f(\alpha(t))$, the arc $\alpha$ belongs to $\mathcal{L}_{a} \cap \mathcal{A}_{k}(f)$ if and only if $\phi(0)$ belongs to $X_{\gamma(a)}$,

$$
F^{\prime}(0)=F^{\prime \prime}(0)=\cdots=F^{\left(k-m_{f}(a)-1\right)}(0)=0, \text { and } F^{\left(k-m_{f}(a)\right)}(0) \neq 0 .
$$

Indeed, they implies that the order of $f \circ \alpha$ is equal to $k$. That system determines the coefficients of $\phi$ (and so of $\alpha$ ), and in particular enables us to compute the measure of $\mathcal{L}_{a} \cap \mathcal{A}_{k}(f)$ in case $k>m_{f}(a)$. Since $F(t)=f_{\gamma(a)}(\phi(t))+R(t)$, we obtain that

$$
F^{\prime}(t)=\sum_{i=1}^{n} \phi_{i}^{\prime}(t) \frac{\partial f_{\gamma(a)}}{\partial x_{i}}(\phi(t))+R^{\prime}(t) .
$$

Choosing for $\phi(0)$ any point in $X_{\gamma(a)}$, there exists at least one partial derivatives of $f_{\gamma(a)}$, say $\frac{\partial f_{\gamma(a)}}{\partial x_{i_{0}}}$, that does not vanish at $\phi(0)$ because $f$ is non-degenerate. This shows that we can freely choose the remaining coefficients of $\phi$ as soon as we fix the value of $\phi_{i_{0}}^{\prime}(0)$ so that $F^{\prime}(0)=0$. We proceed in a similar way for higher order derivatives of $F$. The computation of $F^{(2)}$ gives

$$
\begin{aligned}
F^{(2)}(t) & =\sum_{i=1}^{n} \phi_{i}^{(2)}(t) \frac{\partial f_{\gamma(a)}}{\partial x_{i}}(\phi(t))+\sum_{i, j=1}^{n} \phi_{i}^{\prime}(t) \phi_{j}^{\prime}(t) \frac{\partial^{2} f_{\gamma(a)}}{\partial x_{i} \partial x_{j}}(\phi(t))+R^{(2)}(t) \\
& =\sum_{i=1}^{n} \phi_{i}^{(2)}(t) \frac{\partial f_{\gamma(a)}}{\partial x_{i}}(\phi(t))+R_{2}(t)
\end{aligned}
$$

where $R_{2}(t)$ is equal to the sum of the last two terms in the first line. Repeating the procedure for any $l \in\left\{1, \ldots, k-m_{f}(a)\right\}$, there exists a power series $R_{l}$ such that

$$
F^{(l)}(t)=\sum_{i=1}^{n} \phi_{i}^{(l)}(t) \frac{\partial f_{\gamma(a)}}{\partial x_{i}}(\phi(t))+R_{l}(t) .
$$

In particular, an arbitrary choice of the coefficients of $\phi$ enables us to solve the system $F^{\prime}(0)=F^{\prime \prime}(0)=\cdots=F^{\left(k-m_{f}(a)-1\right)}(0)=0$ as soon as we fix the values of $\phi_{i_{0}}^{\prime}(0), \ldots, \phi_{i_{0}}^{\left(k-m_{f}(a)-1\right)}(0)$. Finally, to take into account the additional fact $F^{\left(k-m_{f}(a)\right)}(0) \neq 0$ in order to guarantee that $\alpha$ belongs to $\mathcal{L}_{a} \cap \mathcal{A}_{k}(f)$, we simply need to exclude one value for $\phi_{i_{0}}^{\left(k-m_{f}(a)\right)}(0)$. We conclude that the measure of the truncation of $\mathcal{L}_{a} \cap \mathcal{A}_{k}(f)$ stabilizes sufficiently large $k$, and that

$$
p_{k}\left(\mathcal{L}_{a} \cap \mathcal{A}_{k}(f)\right) \simeq X_{\gamma(a)} \times \mathbb{R}^{*} \times \mathbb{R}^{k n-s(a)-\left(k-m_{f}(a)\right)},
$$

so that

$$
\left[\mathcal{L}_{a} \cap \mathcal{A}_{k}(f)\right]=(\mathbb{L}-1)\left[X_{\gamma(a)}\right] \mathbb{L}^{-s(a)-k+m_{f}(a)} .
$$


Remark 2.5. The function $f_{\gamma}$ associated with the face $\gamma$ of the Newton polyhedron of $f$ is said to be not definite if $X_{\gamma} \neq \emptyset$. Set

$$
m_{0}(f)=\min \left\{m_{f}(a): a \in\left(\mathbb{N}^{*}\right)^{n}, f_{\gamma(a)} \text { is not definite }\right\} .
$$

Then Lemma 2.4 (in the case " $k>m_{f}(a)$ ") shows that any integer greater than or equal to $m_{0}(f)$ belongs to the set of Fukui invariant $A(f)$ of $f$. We put $T(f)=$ $\left\{m \in \mathbb{N}: m \geq m_{0}(f)\right\}$, so that $T(f) \subset A(f)$. The remaining integers contained in the set of Fukui invariants coincide with the sets

$$
\begin{aligned}
S(f) & =\left\{m_{f}(a): a \in\left(\mathbb{N}^{*}\right)^{n}, \exists c \in\left(\mathbb{R}^{*}\right)^{n}, f_{\gamma(a)}(c) \neq 0\right\}, \\
S^{ \pm}(f) & =\left\{m_{f}(a): a \in\left(\mathbb{N}^{*}\right)^{n}, \exists c \in\left(\mathbb{R}^{*}\right)^{n}, f_{\gamma(a)}(c)= \pm 1\right\},
\end{aligned}
$$

as illustrated by Lemma 2.4 (in the case " $k=m_{f}(a)$ "). As a consequence

$$
A(f)=S(f) \cup T(f) \text { and } A^{ \pm}(f)=S^{ \pm}(f) \cup T(f) .
$$

Another consequence of Lemma 2.4 is a nice description of the measure of the arc spaces associated with $f$ in terms of the geometry of $f$ and of the combinatorics of the Newton polyhedron of $f$. For a compact face $\gamma$ of $\Gamma_{+}(f)$ and $k \in \mathbb{N}^{*}$, we define elements $P_{k}(\gamma)$ and $Q_{k}(\gamma)$ in the localized Grothendieck ring of real algebraic variety by setting

$$
P_{k}(\gamma)=\sum_{a \in\left(\mathbb{N}^{*}\right)^{n}, \gamma(a)=\gamma, m_{f}(a)=k} \mathbb{L}^{-s(a)}
$$

and

$$
Q_{k}(\gamma)=\sum_{a \in\left(\mathbb{N}^{*}\right)^{n}, \gamma(a)=\gamma, m_{f}(a)<k} \mathbb{L}^{-k+m_{f}(a)-s(a)} .
$$

Theorem 2.6. Let $k \in \mathbb{N}^{*}$. If $f$ is a non-degenerate polynomial, the measure of the arcs spaces associated with $f$ can be decomposed into several terms associated with the compact faces of $\Gamma_{+}(f)$ as

$$
\left[\mathcal{A}_{k}(f)\right]=\sum_{\gamma<\Gamma(f)}\left((\mathbb{L}-1)^{n}-\left[X_{\gamma}\right]\right) P_{k}(\gamma)+(\mathbb{L}-1) \sum_{\gamma<\Gamma(f)}\left[X_{\gamma}\right] Q_{k}(\gamma)
$$

In the case with sign, we obtain similarly

$$
\left[\mathcal{A}_{k}^{ \pm}(f)\right]=\sum_{\gamma<\Gamma(f)}\left[X_{\gamma}^{ \pm}\right] P_{k}(\gamma)+\sum_{\gamma<\Gamma(f)}\left[X_{\gamma}\right] Q_{k}(\gamma)
$$

Remark 2.7. This decomposition of the measure of the arc spaces $\mathcal{A}_{k}(f)$ and $\mathcal{A}_{k}^{ \pm}(f)$, where $k \in \mathbb{N}^{*}$, into a sum of two terms is motivated by the difference between:

- $\operatorname{arcs}$ of order $a \in\left(\mathbb{N}^{*}\right)^{n}$ that directly contribute to the coefficient of $t^{k}$, i.e. with $k=m_{f}(a)$, and

- $\operatorname{arcs}$ of order $a \in\left(\mathbb{N}^{*}\right)^{n}$ that contribute to a term of order $k>m_{f}(a)$.

Understanding both contributions will be the main step in section 3.3 in order to recover the weights of a weighted homogeneous polynomial from the zeta functions. 
Remark 2.8. Let us rewrite $P_{k}(\gamma)$ as

$$
P_{k}(\gamma)=\sum_{a \in\left(\mathbb{N}^{*}\right)^{n}, \gamma(a)=\gamma, m_{f}(a)=k} \mathbb{L}^{-k+m_{f}(a)-s(a)},
$$

since $m_{f}(a)$ is equal to $k$ for those $a$ appearing in the summation. Using this expression of $P_{k}(\gamma)$, we see that the difference between $P_{k}(\gamma)$ and $Q_{k}(\gamma)$ lies in the value of $m_{f}$ at $a \in\left(\mathbb{N}^{*}\right)^{n}$. In particular, in order to understand the powers of $\mathbb{L}$ in the expressions of $P_{k}(\gamma)$ and $Q_{k}(\gamma)$, we are lead to focus on the levels of the piecewise linear function $m_{f}-s$ defined on the dual of the Newton polyhedron of $f$, and more precisely on the subsets defined by $m_{f}=k$ and $m_{f}<k$ for a given integer $k \in \mathbb{N}^{*}$. In the sequel, we denote by $h$ the function $h=m_{f}-s$.

Proof of Theorem 2.6. We decompose the measure of $\mathcal{A}_{k}(f)$ with respect to the order of the arcs and reconstitute it with respect to the belonging of these orders to the different compact faces of the Newton Polyhedron of $f$. First, it follows from the computation in Example 1.1 that

$$
\left[\mathcal{A}_{k}(f)\right]=\sum_{a \in\left(\mathbb{N}^{*}\right)^{n}}\left[\mathcal{L}_{a} \cap \mathcal{A}_{k}(f)\right]
$$

since the measure of $\left[\mathcal{L}_{a} \cap \mathcal{A}_{k}(f)\right]$ is equal to zero for $a \in \mathbb{N}^{n} \backslash\left(\mathbb{N}^{*}\right)^{n}$. Therefore, we obtain from Lemma 2.4 that

$$
\begin{aligned}
{\left[\mathcal{A}_{k}(f)\right]=} & \sum_{\gamma<\Gamma(f)}\left((\mathbb{L}-1)^{n}-\left[X_{\gamma}\right]\right) \sum_{a \in\left(\mathbb{N}^{*}\right)^{n}, \gamma(a)=\gamma, m_{f}(a)=k} \mathbb{L}^{-s(a)} \\
& +(\mathbb{L}-1) \sum_{\gamma<\Gamma(f)}\left[X_{\gamma}\right] \sum_{a \in\left(\mathbb{N}^{*}\right)^{n}, \gamma(a)=\gamma, m_{f}(a)<k} \mathbb{L}^{-k+m_{f}(a)-s(a)} .
\end{aligned}
$$

Similarly, in the case with sign, we obtain that

$$
\begin{aligned}
{\left[\mathcal{A}_{k}^{ \pm}(f)\right]=} & \sum_{a \in\left(\mathbb{N}^{*}\right)^{n}}\left[\mathcal{L}_{a} \cap \mathcal{A}_{k}^{ \pm}(f)\right] \\
= & \sum_{\gamma<\Gamma(f)}\left[X_{\gamma}^{ \pm}\right] \sum_{a \in\left(\mathbb{N}^{*}\right)^{n}, \gamma(a)=\gamma, m_{f}(a)=k} \mathbb{L}^{-s(a)} \\
& +\sum_{\gamma<\Gamma(f)}\left[X_{\gamma}\right] \sum_{a \in\left(\mathbb{N}^{*}\right)^{n}, \gamma(a)=\gamma, m_{f}(a)<k} \mathbb{L}^{-k+m_{f}(a)-s(a)} .
\end{aligned}
$$

\section{Estimate of Degrees}

This section is the heart of the paper. We give a linear bound for the degree of the virtual Poincaré polynomial of the arc spaces $\mathcal{A}_{k}(f)$ and $\mathcal{A}_{k}^{ \pm}(f)$ associated with a given polynomial $f$, and we investigate when this bound is sharp, in the sense that the equality holds for infinitely many $k$. Using this bound, we define the leading exponent of $\beta\left(\mathcal{A}_{k}(f)\right)$; this leading exponent is encoded in the zeta function of $f$.

Recall that $u$ stands for the Poincaré polynomial of the affine line. In the sequel the notation $P_{k}(\gamma)$ and $Q_{k}(\gamma)$ introduced for Theorem 2.6 will be interpreted with the virtual Poincaré polynomial rather than the measure in the localised Grothendieck ring (namely $\mathbb{L}$ is replaced by $u$ ).

By convention, we define $\operatorname{dim} \emptyset=-\infty$ and $\max \emptyset=-\infty$. 
3.1. Notation. We are going to prepare several pieces of notation continuing those introduced in section 2.1. Since $m_{f}(a)=\min \left\{\langle a, \nu\rangle: \nu \in \Gamma_{+}(f)\right\}$ for $a \in \mathbb{R}_{+}^{n}$, the smallest component of $a \in \mathbb{R}_{+}^{n}$ is equal to zero if $m_{f}(a)=0$. This means that if $a$ belongs to $\left(\mathbb{R}_{+}^{*}\right)^{n}$, then $m_{f}(a)>0$. We define $e_{f}$ by

$$
e_{f}=\sup \left\{1-\frac{s(a)}{m_{f}(a)}: a \in\left(\mathbb{R}_{+}^{*}\right)^{n}\right\} \text {. }
$$

The number $e_{f}$ will enable us to give a bound for the degree of the virtual Poincaré polynomial of the arc spaces. Finaly, we denote by $\Lambda_{\max }(f) \subset \mathbb{N}^{n}$ the primitive generators of 1 -cones in $\Gamma^{*}(f)$ that realise $e_{f}$; that is,

$$
\Lambda_{\max }(f)=\left\{v \in \Lambda(f): m_{f}(v)>0, e_{f}=1-\frac{s(v)}{m_{f}(v)}\right\} .
$$

Remark 3.1. If $f$ is convenient, then the facets are supported by primitive vectors $v$ with $v \in\left(\mathbb{N}^{*}\right)^{n}$ or $e_{i}(i=1, \ldots, n)$. Since $m_{f}\left(e_{i}\right)=0$, we conclude that $\Lambda_{\max }(f) \subset$ $\left(\mathbb{N}^{*}\right)^{n}$. If $f$ is not convenient, then there are vectors $v \in \Lambda(f) \subset\left(\mathbb{N}^{n} \backslash\left(\mathbb{N}^{*}\right)^{n}\right)$ with $m(v)>0$. These $v$ support non-compact facets. Sometimes such $v$ belongs to $\Lambda_{\max }(f)$ and it may happen that $\Lambda_{\max }(f)$ only contains such elements. Thus, the set $\Lambda_{\max }(f) \cap\left(\mathbb{N}^{*}\right)^{n}$ could be empty.

Example 3.2. For the two variable function $f(x, y)=x^{5}+x^{2} y$, the set of primitive generators of 1 -cones in $\Gamma^{*}(f)$ is $\Lambda(f)=\{(1,0),(1,3),(0,1)\}$. The number $e_{f}$ is equal to $1 / 2$ and $\Lambda_{\max }(f)=\{(1,0)\}$.

Example 3.3. If $f(x, y, z)=x y^{d}+y z^{d}+z x^{d}$, we have

$$
\Lambda(f)=\{(1,0,0),(0,1,0),(0,0,1),(1, d, 0),(0,1, d),(d, 0,1),(1,1,1)\}
$$

and $e_{f}=1-\min \left\{\frac{d+1}{d}, \frac{3}{d+1}\right\}=1-\frac{3}{d+1}$. So we obtain that $\Lambda_{\max }(f)=\{(1,1,1)\}$.

Example 3.4. For $f=\left(x^{3}+y^{3}\right) z+x^{d}+y^{d}(d \geq 4)$, we have

$$
\Lambda(f)=\{(1,0,0),(0,1,0),(0,0,1),(1,1,0),(1,1, d-3)\}
$$

and $e_{f}=1-\min \left\{\frac{2}{3}, \frac{d-1}{d}\right\}=1-2 / 3=1 / 3$. We thus obtain that $\Lambda_{\max }(f)=\{(1,1,0)\}$.

Proposition 3.5. For any integer $k \in \mathbb{N}^{*}$, we have that

$$
\begin{aligned}
e_{f} & =\sup \left\{1-\frac{s(a)}{m_{f}(a)}: a \in\left(\mathbb{R}_{+}^{*}\right)^{n}, m_{f}(a)=k\right\} \\
& =\max \left\{1-\frac{s(a)}{m_{f}(a)}: a \in \Lambda(f), m_{f}(a)>0\right\} .
\end{aligned}
$$

In particular $e_{f} \in \mathbb{Q}$. Moreover

$$
e_{f} \geq \max \left\{1-\frac{s(a)}{m_{f}(a)}: a \in\left(\mathbb{N}^{*}\right)^{n}, m_{f}(a)=k\right\} .
$$

and the equality holds for infinitely many $k$ if and only if $\Lambda_{\max }(f) \cap\left(\mathbb{N}^{*}\right)^{n} \neq \emptyset$.

Proof of Proposition 3.5. Since the function $s$ is linear and the function $m_{f}$ is piecewise linear, then $\frac{s(\lambda a)}{m_{f}(\lambda a)}=\frac{s(a)}{m_{f}(a)}$ for any $\lambda \in \mathbb{R}_{+}^{*}$ and $a \in\left(\mathbb{R}_{+}^{*}\right)^{n}$. In particular, the right-hand side of the first equality does not depend on $k$. Then the equality is clearly attained for infinitely many $k$ by definition of $e_{f}$. 
To see the second equality, we choose $k=1$ and we take a sequence $\left(a^{j}\right)_{j \in \mathbb{N}} \in$ $\left(\left(\mathbb{R}_{+}^{*}\right)^{n}\right)^{\mathbb{N}}$ with $m_{f}\left(a^{j}\right)=1$ and $1-s\left(a^{j}\right) \rightarrow e_{f}$ as $j \rightarrow \infty$. The sequence $\left(s\left(a^{j}\right)\right)_{j \in \mathbb{N}}$ is bounded, therefore so is $\left(a^{j}\right)_{j \in \mathbb{N}}$ and, taking a subsequence if necessary, we may assume that the sequence $\left(a^{j}\right)_{j \in \mathbb{N}}$ is convergent to a limit $b \in \mathbb{R}_{+}^{n}$ satisfying $m_{f}(b)=1$ and $e_{f}=1-s(b)$. We want to prove that there exist $\lambda \in \mathbb{R}_{+}^{*}$ and a primitive generator $v \in \Lambda(f)$ with $m_{f}(\lambda v)=1$ and $s(\lambda v)=s(b)$. Assume $b$ is not such a multiple of an element in $\Lambda(f)$. Denote by $\sigma$ the subcone of $\Gamma^{*}(f)$ corresponding to the face $\gamma_{f}(b)$ of the Newton polyhedron of $f$, and consider the restriction of $s$ to $\sigma \cap\left\{m_{f}=1\right\}$. The restriction of the linear function $s$ must be constant otherwise $e_{f}=1-s(b)$ can not be the supremum of $1-s / m$. As a consequence, for any $v \in \Lambda(f)$ with $v \in \sigma$. and for $\lambda \in \mathbb{R}_{+}^{*}$ with $m_{f}(\lambda v)=1$, we obtain $s(\lambda v)=s(b)$.

The last inequality is clear from the first equality, and the condition on when the equality holds comes from the second equality.

By definition, $e_{f}$ is strictly less than 1 . We describe its sign in next proposition.

Proposition 3.6. The sign of $e_{f}$ is decided by the following conditions.

- If $(1, \ldots, 1) \notin \Gamma_{+}(f)$, then $e_{f}>0$.

- If $(1, \ldots, 1) \in \Gamma(f)$, then $e_{f}=0$.

- If $(1, \ldots, 1)$ is in the interior of $\Gamma_{+}(f)$, then $e_{f}<0$.

Proof. By definition of the function $m_{f}$, the following equivalences hold:

- $(1, \ldots, 1) \in \Gamma_{+}(f)$ if and only if $m_{f}(a) \leq s(a)$ for any $a \in\left(\mathbb{R}_{+}^{*}\right)^{n}$.

- $(1, \ldots, 1) \in$ the interior of $\Gamma_{+}(f)$ if and only if $m_{f}(a)<s(a)$ for any $a \in$ $\left(\mathbb{R}_{+}^{*}\right)^{n}$

- $(1, \ldots, 1) \notin \Gamma_{+}(f)$ if and only if $m_{f}(a)>s(a)$ for some $a \in\left(\mathbb{R}_{+}^{*}\right)^{n}$.

To prepare for the forthcoming proof of Theorem 3.10, we introduce some more notation corresponding to numbers $e_{\gamma}$ analogous to $e_{f}$ but restricted to a given face $\gamma$ of the Newton polyhedron of $f$, and to elements $a \in\left(\mathbb{R}_{+}^{*}\right)^{n}$ realising such $e_{\gamma}$.

Definition 3.7. Let $\gamma$ be a compact face of $\Gamma_{+}(f)$. We set

$$
\begin{aligned}
e_{\gamma} & =\sup \left\{1-\frac{s(a)}{m_{f}(a)}: a \in\left(\mathbb{R}_{+}^{*}\right)^{n}, \gamma(a)=\gamma\right\}, \\
m_{\gamma} & =\min \left\{m_{f}(a): a \in\left(\mathbb{N}^{*}\right)^{n}, \gamma(a)=\gamma\right\} .
\end{aligned}
$$

For $k \in \mathbb{N}^{*}$, we denote by $E_{k}$ the set of $n$-tuples of positive numbers realising $e_{f}$ on the level $m_{f}=k$,

$$
E_{k}=\left\{a \in\left(\mathbb{R}_{+}^{*}\right)^{n}: e_{f}=1-\frac{s(a)}{m_{f}(a)}, m_{f}(a)=k\right\},
$$

and we denote by $E_{k}(\gamma)$ its version relative to the face $\gamma$; that is,

$$
E_{k}(\gamma)=\left\{a \in\left(\mathbb{R}_{+}^{*}\right)^{n}: e_{\gamma}=1-\frac{s(a)}{m_{f}(a)}, m_{f}(a)=k, \gamma(a)=\gamma\right\} .
$$

Also, we define $e_{f}^{0}$ by

$$
e_{f}^{0}=\max \left\{m_{\gamma} e_{\gamma}: X_{\gamma} \neq \emptyset\right\}
$$


Similarly in the case with sign, we set

$$
e_{f}^{ \pm}=\max \left\{e_{\gamma}: X_{\gamma}^{ \pm} \neq \emptyset\right\} .
$$

Remark 3.8. We have $e_{\gamma}=\max \left\{1-\frac{s(a)}{m_{f}(a)}: a \in \Lambda(f), \gamma(a) \supset \gamma\right\}$ as a consequence of the proof of Proposition 3.5. It is clear that $e_{f}=\max \left\{e_{\gamma}: \gamma<\Gamma_{+}(f)\right\}$.

For $k \in \mathbb{N}^{*}$ and $\gamma$ a compact face of $\Gamma_{+}(f)$, the set $E_{k}(\gamma)$ is defined by a linear equation in the cone corresponding to $\gamma$, so $E_{k}(\gamma)$ is convex. In fact, the same holds true for $E_{k}$.

Lemma 3.9. For $k \in \mathbb{N}^{*}$, the set $E_{k}$ is convex.

Proof. Take $a, b \in E_{k}$, and for $t \in[0,1]$ we define $c=(1-t) a+t b$. We have $s(c)=k\left(1-e_{f}\right)$ by linearity of $s$ since $s(a)=s(b)=k\left(1-e_{f}\right)$. We want to show that $m_{f}(c)=k$. On one hand, we know that $m_{f}(c) \geq k$ by definition of $m_{f}$. On the other hand, since $m_{f}\left(\frac{k}{m_{f}(c)} c\right)=k$, we have

$$
k\left(1-e_{f}\right) \leq s\left(\frac{k}{m_{f}(c)} c\right)
$$

by definition of $e_{f}$. Moreover

$$
s\left(\frac{k}{m_{f}(c)} c\right)=\frac{k}{m_{f}(c)} s(c)=\frac{k}{m_{f}(c)} k\left(1-e_{f}\right) .
$$

and, since $1-e_{f}>0$, we have $m_{f}(c) \leq k$. Therefore, $m_{f}(c)=k$ and $c$ belongs to $E_{k}$.

3.2. Linear bound for the degree. Let $A_{k}(u)$ denote elements of $\mathbb{Z}[u]\left[\left[u^{-1}\right]\right]$ for $k \in \mathbb{N}^{*}$. We look for a linear bound for the degree of $A_{k}(u)$, namely a bound of the form

$$
\operatorname{deg} A_{k}(u) \leq \alpha k+\beta,
$$

with $(\alpha, \beta) \in \mathbb{Q} \times \mathbb{Q}$ such that equality holds for infinitely many $k \in \mathbb{N}^{*}$. Note that if it exists, such a pair $(\alpha, \beta)$ is unique. Setting

$$
Z(t)=\sum_{k \geq 1} A_{k}(u) t^{k}, \quad A_{k}(u)=c_{k} u^{\alpha k+\beta}+\text { (lower order terms), }
$$

where $c_{k} \in \mathbb{Z}$, we have

$$
\frac{Z\left(u^{-\alpha} t\right)}{u^{\beta}}=\sum_{k \geq 1} \frac{A_{k}(u)}{u^{\alpha k+\beta}} t^{k} \rightarrow \sum_{k \geq 1} c_{k} t^{k} \quad \text { as } u \rightarrow \infty .
$$

So a characterisation of $(\alpha, \beta) \in \mathbb{Q} \times \mathbb{Q}$ is that it is the unique pair such that

$$
\lim _{u \rightarrow \infty} \frac{Z\left(u^{-\alpha} t\right)}{u^{\beta}}
$$

is a non-zero series. For a given integer $k \in \mathbb{N}^{*}$, we call $c_{k} u^{\alpha k+\beta}$ the leading term and $c_{k}$ the leading coefficient with respect to the linear bound of the degree of $A_{k}(u)$. We call $\alpha k+\beta$ the leading exponent of $Z(t)$.

In Theorem 3.10, we give a bound for the degree of the virtual Poincaré polynomials of the arc spaces $\mathcal{A}_{k}(f)$ and $\mathcal{A}_{k}^{ \pm}(f)$ associated with a polynomial function $f$. Moreover, we give a condition so that a linear bound exists for $\beta\left(\mathcal{A}_{k}(f)\right)$. We express these bounds in terms of the integral numbers $e_{f}$, introduced in (3.1), and $e_{f}^{0}$ and $e_{f}^{ \pm}$, introduced in Definition 3.7 . 
Theorem 3.10. Let $f$ be a non-degenerate polynomial. For all $k \in \mathbb{N}^{*}$, the degree bound of the virtual Poincaré polynomial of $\mathcal{A}_{k}(f)$ is given by

$$
\operatorname{deg} \beta\left(\mathcal{A}_{k}(f)\right) \leq \begin{cases}n-k+k e_{f} & \text { if } e_{f}>0, \\ n-k & \text { if } e_{f}=0, \\ n-k+\max \left\{k e_{f}, e_{f}^{0}\right\} & \text { if } e_{f}<0 .\end{cases}
$$

Moreover if $e_{f} \geq 0$, these bounds give the leading exponent of $Z_{f}(t)$ if and only if $\Lambda_{\max }(f) \cap\left(\mathbb{N}^{*}\right)^{n} \neq \emptyset$. In the case with sign, we have

$$
\operatorname{deg} \beta\left(\mathcal{A}_{k}^{ \pm}(f)\right) \leq \begin{cases}n-1-k+k e_{f}^{ \pm} & \text {if } e_{f}^{ \pm}>0, \\ n-1-k & \text { if } e_{f}^{ \pm}=0, \\ n-1-k+\max \left\{k e_{f}^{ \pm}, e_{f}^{0}\right\} & \text { if } e_{f}^{ \pm}<0 .\end{cases}
$$

Proof. The equalities in Theorem 2.6 imply that

$$
\operatorname{deg} \beta\left(\mathcal{A}_{k}(f)\right)=\max \left\{p_{k}, q_{k}\right\},
$$

where

$$
\begin{aligned}
p_{k} & =n+\max \left\{-s(a): a \in\left(\mathbb{N}^{*}\right)^{n}, m_{f}(a)=k\right\}, \\
q_{k} & =1+\max \left\{q_{k}(\gamma): X_{\gamma} \neq \emptyset, \gamma<\Gamma(f)\right\}, \\
q_{k}(\gamma) & =\operatorname{dim} X_{\gamma}+\max \left\{-s(a)+m_{f}(a)-k: a \in\left(\mathbb{N}^{*}\right)^{n}, m_{f}(a)<k, \gamma(a)=\gamma\right\} .
\end{aligned}
$$

Concerning the term $p_{k}$, we have

$$
\begin{aligned}
p_{k} & =n-k+\max \left\{m_{f}(a)-s(a): a \in\left(\mathbb{N}^{*}\right)^{n}, m_{f}(a)=k\right\} \\
& =n-k+k \max \left\{1-\frac{s(a)}{m_{f}(a)}: a \in\left(\mathbb{N}^{*}\right)^{n}, m_{f}(a)=k\right\} \\
& \leq n-k+k e_{f},
\end{aligned}
$$

and equality holds for infinitely many $k$ if and only if $\Lambda_{\max }(f) \cap\left(\mathbb{N}^{*}\right)^{n} \neq \emptyset$, by Proposition 3.5. Concerning the term $q_{k}$, consider a face $\gamma<\Gamma(f)$. Then

$$
\begin{aligned}
q_{k}(\gamma) & =\operatorname{dim} X_{\gamma}-k+\sup \left\{m_{f}(a)-s(a): a \in\left(\mathbb{N}^{*}\right)^{n}, m_{f}(a)<k, \gamma(a)=\gamma\right\} \\
& =\operatorname{dim} X_{\gamma}-k+\sup \left\{m_{f}(a)\left(1-\frac{s(a)}{m_{f}(a)}\right): a \in\left(\mathbb{N}^{*}\right)^{n}, m_{f}(a)<k, \gamma(a)=\gamma\right\} .
\end{aligned}
$$

Here we remark that if $e_{f}>0$, then

$$
q_{k}(\gamma)<n-1-k+k e_{\gamma}
$$

whilst if $e_{f}=0$, then

$$
q_{k}(\gamma) \leq n-1-k
$$

Finally, in the case $e_{f} \leq 0$, we obtain

$$
q_{k}(\gamma) \leq n-1-k+m_{\gamma} e_{\gamma}
$$

and the inequality is not optimal in general. Finally for $q_{k}$ the bound is

$$
q_{k} \leq \begin{cases}n-k-1+k e_{f} & \text { if } e_{f}>0 \\ n-k & \text { if } e_{f}=0 \\ n-k+\max \left\{m_{\gamma} e_{\gamma}: X_{\gamma} \neq \emptyset\right\} & \text { if } e_{f} \leq 0\end{cases}
$$


As a consequence

$$
\operatorname{deg} \beta\left(\mathcal{A}_{k}(f)\right)=\sup \left\{p_{k}, q_{k}\right\} \leq \begin{cases}n-k+k e_{f} & \text { if } e_{f} \geq 0 \\ n-k+\max \left\{k e_{f}, m_{\gamma} e_{\gamma}: X_{\gamma} \neq \emptyset\right\} & \text { if } e_{f}<0 .\end{cases}
$$

In the case with sign, we obtain similarly by Theorem 2.6

$$
\operatorname{deg} \beta\left(\mathcal{A}_{k}^{ \pm}(f)\right)=\sup \left\{p_{k}^{ \pm}(\gamma): X_{\gamma}^{ \pm} \neq \emptyset, q_{k}(\gamma): X_{\gamma} \neq \emptyset ; \gamma<\Gamma_{+}(f)\right\}
$$

where

$$
p_{k}^{ \pm}(\gamma)=\operatorname{dim} X_{\gamma}^{ \pm}+\sup \left\{-s(a): a \in\left(\mathbb{N}^{*}\right)^{n}, m_{f}(a)=k, \gamma(a)=\gamma\right\} .
$$

Then

$$
\begin{aligned}
p_{k}^{ \pm}(\gamma) & =\operatorname{dim} X_{\gamma}^{ \pm}-k+\sup \left\{m_{f}(a)-s(a): a \in\left(\mathbb{N}^{*}\right)^{n}, m_{f}(a)=k, \gamma(a)=\gamma\right\} \\
& =\operatorname{dim} X_{\gamma}^{ \pm}-k+k \sup \left\{1-\frac{s(a)}{m_{f}(a)}: a \in\left(\mathbb{N}^{*}\right)^{n}, m_{f}(a)=k, \gamma(a)=\gamma\right\} \\
& \leq \operatorname{dim} X_{\gamma}^{ \pm}-k+k e_{\gamma},
\end{aligned}
$$

and equality is attained whenever $E_{k}(\gamma) \cap\left(\mathbb{N}^{*}\right)^{n} \neq \emptyset$. As a consequence

$$
\begin{aligned}
& \operatorname{deg} \beta\left(\mathcal{A}_{k}^{ \pm}(f)\right)=\sup \left\{p_{k}^{ \pm}(\gamma): X_{\gamma}^{ \pm} \neq \emptyset, q_{k}(\gamma): X_{\gamma} \neq \emptyset\right\} \\
& \leq \begin{cases}n-1-k+k \max \left\{e_{\gamma}: X_{\gamma}^{ \pm} \neq \emptyset\right\} & \text { if } e_{f}^{ \pm}>0 \\
n-1-k+\max \left\{k e_{\gamma}: X_{\gamma}^{ \pm} \neq \emptyset, m_{\gamma} e_{\gamma}: X_{\gamma} \neq \emptyset\right\} & \text { if } e_{f}^{ \pm} \leq 0,\end{cases}
\end{aligned}
$$

and the proof is completed.

In the cases that the bounds above do not give the leading exponent, we conclude some information on the degree on the arc spaces. In fact, we have the following results.

Proposition 3.11. If $e_{f} \geq 0$, then

$$
e_{f}=1+\limsup _{k \rightarrow+\infty} \frac{\operatorname{deg} \beta\left(\mathcal{A}_{k}\right)}{k} .
$$

Proof. The result follows directly from Theorem 3.10 if $\Lambda_{\max }(f) \cap\left(\mathbb{N}^{*}\right)^{n} \neq \emptyset$. Otherwise, we know that for $k \in \mathbb{N}^{*}$ and $\epsilon>0$, there exist $a \in\left(\mathbb{Q}^{*}\right)^{n}$ with

$$
0 \leq e_{f}-\left(1-\frac{s(a)}{m_{f}(a)}\right) \leq \epsilon
$$

by definition of $e_{f}$. In particular, there exists sufficientlly large $k_{\epsilon}$ such that for $k \in \mathbb{N}^{*}$ a multiple of $k_{\epsilon}$, there exist $a \in\left(\mathbb{N}^{*}\right)^{n}$ such that the inequality above is satisfied together with $m_{f}(a)=k$. As a consequence, using the notation introduced in the proof of Theorem 3.10, we have

$$
p_{k} \geq n-k+k\left(e_{f}-\epsilon\right)
$$

for $k$ a multiple of $k_{\epsilon}$. This implies that

$$
n-k+k\left(e_{f}-\epsilon\right) \leq \operatorname{deg} \beta\left(\mathcal{A}_{k}\right) \leq n-k+k e_{f},
$$

and thus

for $k$ a multiple of $k_{\epsilon}$.

$$
e_{f}-\epsilon \leq \frac{\operatorname{deg} \beta\left(\mathcal{A}_{k}\right)-n+k}{k} \leq e_{f}
$$


As a consequence of Theorem 3.10 and Proposition 3.11, we obtain the following result.

Theorem 3.12. Let $f$ be a finitely determined non-degenerate polynomial with $e_{f} \geq$ 0. Then

$$
\operatorname{deg} \beta\left(\mathcal{A}_{k}(f)\right) \leq n-k+k e_{f}
$$

and the bound is attained by infinitely many $k \in \mathbb{N}$.

Proof. Since $f$ is finitely determined, then $f$ and $g=f+\sum_{i=1}^{n} x_{i}^{d}$ are right-equivalent for $d \in \mathbb{N}$ big enough. In particular $f$ and $g$ share the same zeta functions, and by Proposition 3.11 we obtain $e_{f}=e_{g}$. Since $g$ is convenient, we know that $\Lambda_{\max }(g) \cap$ $\left(\mathbb{N}^{*}\right)^{n} \neq \emptyset$ and therefore the linear bound

$$
\operatorname{deg} \beta\left(\mathcal{A}_{k}(g)\right) \leq n-k+k e_{g}
$$

is attained for infinitely many $k \in \mathbb{N}$ by Theorem 3.10. This means that the linear bound

$$
\operatorname{deg} \beta\left(\mathcal{A}_{k}(f)\right) \leq n-k+k e_{f}
$$

is attained by infinitely many $k \in \mathbb{N}$.

In the case $e_{f} \leq 0$, this superior limit in Theorem 3.10 also provides some information.

Proposition 3.13. In the case $e_{f}<0$, we have

$$
1+\limsup _{k \rightarrow+\infty} \frac{\operatorname{deg} \beta\left(\mathcal{A}_{k}\right)}{k} \in\left[\max \left\{e_{\gamma}: X_{\gamma} \neq \emptyset\right\}, 0\right],
$$

and it is equal to $e_{f}$ if $\{f=0\}=\{0\}$.

Proof. If $\{f=0\}=\{0\}$, then the degree of the virtual Poincaré polynomial of $\mathcal{A}_{k}(f)$ is given by $p_{k}$, using the notation introduced in the proof of Theorem 3.10, and the same proof as that of Proposition 3.11 gives the result.

If there exists a face $\gamma$ with $X_{\gamma} \neq \emptyset$, then

$$
\frac{\operatorname{deg} \beta\left(\mathcal{A}_{k}\right)-n+k}{k} \leq \frac{\max \left\{k e_{f}, e_{f}^{0}\right\}}{k}
$$

by Theorem 3.10. Moreover the right-hand side is bounded by $e_{f}^{0} / k$ for $k$ sufficiently large since $e_{f}<0$, so that the superior limit is less than or equal to zero. Now, choose $\epsilon>0$. Similarly to the proof of Proposition 3.11, there exist $k_{\epsilon} \in \mathbb{N}^{*}$ and $a \in\left(\mathbb{N}^{*}\right)^{n}$ such that

$$
0 \leq e_{f}-\left(1-\frac{s(a)}{m_{f}(a)}\right) \leq \epsilon
$$

and $m_{f}(a)=k_{\epsilon}$. In particular, for any $k$ equal to one plus a multiple of $k_{\epsilon}$, we have

$$
q_{k}(\gamma) \geq \operatorname{dim} X_{\gamma}-k+(k-1)\left(e_{\gamma}-\epsilon\right) .
$$

As a consequence, for such $k$ we have

$$
\frac{1+\operatorname{dim} X_{\gamma}-n}{k}+\frac{k-1}{k}\left(e_{\gamma}-\epsilon\right) \leq \frac{\operatorname{deg} \beta\left(\mathcal{A}_{k}\right)-n+k}{k}
$$


MOTIVIC INVARIANTS OF REAL POLYNOMIAL FUNCTIONS AND NEWTON POLYHEDRONS 17 since $\operatorname{deg} \beta\left(\mathcal{A}_{k}\right) \geq q_{k} \geq 1+q_{k}(\gamma)$. We thus obtain that

$$
e_{\gamma}-\epsilon \leq \limsup _{k \rightarrow+\infty} \frac{\operatorname{deg} \beta\left(\mathcal{A}_{k}\right)-n+k}{k}
$$

and this concludes the proof.

Next corollary will be useful in the sequel.

Corollary 3.14. Assume that $e_{f} \geq 0$ and $\Lambda_{\max }(f) \cap\left(\mathbb{N}^{*}\right)^{n}$ is a set consisting of one point $v$. Then

$$
\lim _{u \rightarrow+\infty} \frac{Z_{f}\left(u^{1-e_{f}} t\right)}{u^{n}}=\frac{t^{m_{f}(v)}}{1-t^{m_{f}(v)}} .
$$

Proof. By Theorem 3.10, the zeta function of $f$ admits as leading exponent $\left(e_{f}-\right.$ $1) k+n$, and the bound is attained for all multiples of $m_{f}(v)$. In this case the leading coefficient is equal to one, and this implies the result.

A facet is an $(n-1)$-dimensional face of $\Gamma_{+}(f)$. Let $\gamma$ be a compact facet of $\Gamma_{+}(f)$. The associated cone of the subdivision of $\Gamma^{*}(f)$ is one dimensional, and we denote by $v$ in this section the primitive vector with $\gamma(v)=\gamma$. Recall that $h=m_{f}-s$.

Lemma 3.15. For $k \in \mathbb{N}^{*}$, the degree of $P_{k}(\gamma)$ is given by

$$
\operatorname{deg} P_{k}(\gamma)= \begin{cases}-\infty & \text { if } m_{f}(v) \nmid k \\ -k \frac{s(v)}{m_{f}(v)} & \text { if } m_{f}(v) \mid k\end{cases}
$$

and the degree of $Q_{k}(\gamma)$ by

$$
\operatorname{deg} Q_{k}(\gamma)= \begin{cases}-\infty & \text { if } k \leq m_{f}(v), \\ -k+\left\lfloor\frac{k-1}{m_{f}(v)}\right\rfloor\left(m_{f}(v)-s(v)\right) & \text { if } k>m_{f}(v) \text { and } h(v)>0 \\ -k+m_{f}(v)-s(v) & \text { if } k>m_{f}(v) \text { and } h(v) \leq 0\end{cases}
$$

Proof. The proof is a direct consequence of the following equalities:

$$
\begin{aligned}
& \operatorname{deg} P_{k}(\gamma)=\max \left\{-s(a): a=\lambda v \lambda \in \mathbb{N}^{*}, m_{f}(a)=k\right\} \\
& \operatorname{deg} Q_{k}(\gamma)=-k+\max \left\{m_{f}(a)-s(a): a=\lambda v, \lambda \in \mathbb{N}^{*}, m_{f}(\lambda v) \leq k-1\right\},
\end{aligned}
$$

coming from the definition of $P_{k}(\gamma)$ and $Q_{k}(\gamma)$.

In particular, we note that for $k=m_{f}(v)$ we have $\operatorname{deg} P_{k}(\gamma)=-s(v)$ whereas $Q_{k}(\gamma)=0$.

Corollary 3.16. Let $k \in \mathbb{N}^{*}$. Assume that $k$ is divisible by $m_{f}(v)$. Then the sign of $h(v)$ determines the following:

- if $h(v)>0$, then $\operatorname{deg} P_{k}(\gamma)>\operatorname{deg} Q_{k}(\gamma)$,

- if $h(v)=0$, then $\operatorname{deg} P_{k}(\gamma)=\operatorname{deg} Q_{k}(\gamma)$,

- if $h(v)<0$ and $k \neq m_{f}(v)$, then $\operatorname{deg} P_{k}(\gamma)<\operatorname{deg} Q_{k}(\gamma)$.

Proof. If $m_{f}(v)-s(v)>0$ and $m_{f}(v) \mid k$, we obtain

$$
\begin{aligned}
\operatorname{deg} P_{k}(\gamma)-\operatorname{deg} Q_{k}(\gamma) & =-k \frac{s(v)}{m_{f}(v)}+k-\left\lfloor\frac{k-1}{m_{f}(v)}\right\rfloor\left(m_{f}(v)-s(v)\right) \\
& =\left(\frac{k}{m_{f}(v)}-\left\lfloor\frac{k-1}{m_{f}(v)}\right\rfloor\right)\left(m_{f}(v)-s(v)\right)>0
\end{aligned}
$$


and this proves the first statement. If $m_{f}(v)-s(v) \leq 0$ and $m_{f}(v) \mid k$, we obtain

$$
\begin{aligned}
\operatorname{deg} P_{k}(\gamma)-\operatorname{deg} Q_{k}(\gamma) & =-k \frac{s(v)}{m_{f}(v)}+k-\left(m_{f}(v)-s(v)\right) \\
& =\left(\frac{k}{m_{f}(v)}-1\right)\left(m_{f}(v)-s(v)\right),
\end{aligned}
$$

and this implies the remaining cases.

3.3. Convenient weighted homogeneous polynomials. Let $f \in \mathbb{R}\left[x_{1}, \ldots, x_{n}\right]$ be a weighted homogeneous polynomial with respect to the weight $\left(w_{1}, \ldots, w_{n} ; d\right)$. We assume that $f$ is convenient.

In this case $\Gamma_{+}(f)$ has a unique compact facet $\gamma_{f}$, and its associated 1-cone is generated by the primitive vector $v=\left(w_{1}, \ldots, w_{n}\right)$, with $m_{f}(v)=d$.

Note that $\Lambda_{\max }(f)=\{v\} \in\left(\mathbb{N}^{*}\right)^{n}$. As a consequence,

$$
h(v)=m_{f}(v)-\sum_{i=1}^{n} w_{i}
$$

and

$$
e_{f}=\frac{h(v)}{m_{f}(v)}=1-\frac{1}{m_{f}(v)} \sum_{i=1}^{n} w_{i}
$$

In particular, if we are able to compute $h(v)$ and $m_{f}(v)$ from the zeta function, then we can recover the sum of the weights of $f$.

Assume that the zeta function of $f$ is given. Then we can decide whether $e_{f}>0$ of $e_{f} \leq 0$ using Proposition 3.11 and Proposition 3.13. If $e_{f}>0$, we recover the value of $e_{f}$ by Proposition 3.11, together with $m_{f}(v)$ by Corollary 3.14. In this case we derive the value of $h(v)$ as $h(v)=m_{f}(v) e_{f}$.

In case $e_{f} \leq 0$, the situation is more subtle to analyse. Note that if $X_{\gamma_{f}}$ is not empty, then the degree of the virtual Poincaré polynomial of $\mathcal{A}_{k}(f)$ is given by

$$
\left.n+\max \left\{\operatorname{deg} P_{k}\left(\gamma_{f}\right), \operatorname{deg} Q_{k}\left(\gamma_{f}\right)\right\}\right\}
$$

- but it may be less if $X_{\gamma_{f}}$ is empty. The degree of $P_{k}\left(\gamma_{f}\right)$ and $Q_{k}\left(\gamma_{f}\right)$ may be expressed by the formulae

$$
\operatorname{deg} P_{k}\left(\gamma_{f}\right)=-k+\max \left\{h(a): a \in\left(\mathbb{N}^{*}\right)^{n}, m_{f}(a)=k, \gamma(a)=\gamma_{f}\right\}
$$

and

$$
\operatorname{deg} Q_{k}\left(\gamma_{f}\right)=-k+\max \left\{h(a): a \in\left(\mathbb{N}^{*}\right)^{n}, m_{f}(a)<k, \gamma(a)=\gamma_{f}\right\} .
$$

Therefore we are led to analyse the levels of the function $h=m_{f}-s$ on $\mathbb{N}^{n}$, and more precisely on the subsets of $\mathbb{N}^{n}$ defined by $\left\{m_{f}(a)=k\right\}$ and $\left\{m_{f}(a)<k\right\}$.

To begin with, let us forget that we are interested in integral points and describe its levels on $\mathbb{R}_{+}^{n}$. The function $h$ is linear on each cone of the subdivision of $\Gamma^{*}(f)$, therefore its levels are completely described by its value on $v$ and on the canonical basis $\left\{e_{1}, \ldots, e_{n}\right\}$ of $\mathbb{R}^{n}$. Note that $h\left(e_{i}\right)=m_{f}\left(e_{i}\right)-1=-1$ since the Newton polyhedron is convenient.

In particular, the levels of $h$ on $\mathbb{R}_{+}^{n}$ are described as follows:

- if $h(v)=0$, there are only non-positive levels that are cylinders parallel to the line generated by $v$, 
- if $h(v)<0$, there are only strictly negative levels that are unions of $n$ bounded simplexes with a vertex on the line generated by $v$ and other vertexes on $(n-1)$ positive coordinates axis, whereas

- if $h(v)>0$, the levels of $h$ are unions of $n$ unbounded simplexes with a vertex on the line generated by $v$.

Returning to the computation of the degrees of $P_{k}(\gamma)$ and $Q_{k}(\gamma)$, we need to investigate the integral points on these levels.

Lemma 3.17. Let $k \in \mathbb{N}^{*}$. Assume that $e_{f} \leq 0$ and $X_{\gamma_{f}} \neq \emptyset$.

- $\operatorname{deg} \beta\left(\mathcal{A}_{k}(f)\right) \leq n-k$ with equality for infinitely many $k$ if and only if $h(v)=0$,

- $\operatorname{deg} \beta\left(\mathcal{A}_{k}(f)\right)<n-k$ if and only if $h(v)<0$. In this case, for infinitely many $k$, we have

$$
\operatorname{deg} \beta\left(\mathcal{A}_{k}(f)\right)=n-k+\max \left\{h(a): a \in\left(\mathbb{N}^{*}\right)^{n}, X_{\gamma(a)} \neq \emptyset\right\} .
$$

Proof. It suffices to compute the maximum of $h$ on $\left\{a \in\left(\mathbb{R}_{+}\right)^{n}: m_{f}(a)=k\right\}$ and on $\left\{a \in\left(\mathbb{R}_{+}\right)^{n}: m_{f}(a)<k\right\}$.

If $h(v)=0$, then $h$ has non-positive levels. The maximum of $h$ on $\left\{a \in\left(\mathbb{R}_{+}\right)^{n}\right.$ : $\left.m_{f}(a)=k\right\}$ is attained on the line generated by $v$ (in general not at an integral point). In particular, if $k>m_{f}(v)$, the maximum of $h$ on $\left\{a \in\left(\mathbb{R}_{+}\right)^{n}: m_{f}(a)<k\right\}$ is attained at $v$.

In the case $h(v)<0$, then $h$ has strictly negative levels. If $k>m_{f}(v)$, then

$$
h(v) \leq \max \left\{h(a): a \in\left(\mathbb{N}^{*}\right)^{n}, X_{\gamma(a)} \neq \emptyset\right\}<0 .
$$

Remark 3.18. It may happen that $\max \left\{h(a): a \in\left(\mathbb{N}^{*}\right)^{n}\right\}$ is strictly bigger than $h(v)$ in the case $h(v)<0$. Consider for example $f\left(x_{1}, x_{2}, x_{3}\right)=x_{1}^{2}-x_{2}^{2}+x_{3}^{m}$ with $m$ odd. Then $v=(m, m, 2)$ and $m_{f}(v)=2 m$ so that $h(v)=m_{f}(v)-s(v)=$ -2 . However, for $a=(1,1,1)$, we have $h(a)=2-3=-1$ and $\gamma(a)$ is the face corresponding to $x_{1}^{2}-x_{2}^{2}$, so that $X_{\gamma(a)} \neq \emptyset$.

Assume that $e_{f} \leq 0$ and $X_{\gamma_{f}}=\emptyset$. Then $X_{\gamma}=\emptyset$ for all faces $\gamma$ of $\Gamma(f)$, therefore the degree of the virtual Poincaré polynomial of $\mathcal{A}_{k}(f)$ is simply given by the terms $P_{k}(\gamma)$ for $\gamma<\Gamma(f)$, and the discussion becomes easier.

Lemma 3.19. Assume that $e_{f} \leq 0$ and $X_{\gamma_{f}}=\emptyset$. Let $k \in \mathbb{N}^{*}$. Then

- $\operatorname{deg} \beta\left(\mathcal{A}_{k}(f)\right) \leq n-k$ with equality for infinitely many $k$ if and only if $h(v)=0$,

- $\operatorname{deg} \beta\left(\mathcal{A}_{k}(f)\right)<n-k$ if and only if $h(v)<0$.

Proof. The degree of $\beta\left(\mathcal{A}_{k}(f)\right)$ is given by

$$
\max \left\{-s(a): a \in\left(\mathbb{N}^{*}\right)^{n}, m_{f}(a)=k\right\}
$$

because the terms of the form $Q_{k}(\gamma)$ vanish, for $\gamma<\Gamma(f)$. Moreover

$$
\sup \left\{-s(a): a \in\left(\mathbb{R}_{+}\right)^{n}, m_{f}(a)=k\right\}=-s\left(\frac{k}{m_{f}(v)} v\right),
$$


since the levels of $m_{f}$ on $\mathbb{R}_{+}^{n}$ are the boundary of cones with vertex a point lying on the line generated by $v$, whose sides are parallel to the coordinate hyperplanes. As a consequence

$$
\max \left\{-s(a): a \in\left(\mathbb{N}^{*}\right)^{n}, m_{f}(a)=k\right\} \leq-s\left(\frac{k}{m_{f}(v)} v\right),
$$

and the equality holds when $m_{f}(v)$ divides $k$. In particular, $s\left(\frac{k}{m_{f}(v)} v\right)=k$ if $h(v)=0$, whereas $s\left(\frac{k}{m_{f}(v)} v\right)>k$ if $h(v)<0$.

Therefore we are able to recognise the sign of $e_{f}$, and thus the sign of $h(v)=$ $m_{f}(v) e_{f}$, from the zeta function of $f$ via Proposition 3.11 and Proposition 3.13. Moreover we are able to obtain the value of $m_{f}(v)$, and thus of $h(v)$, when we already know that $h(v)$ is greater than or equal to zero thanks to Corollary 3.14. We collect the different possibilities for the sign of $h(v)$ in next proposition.

Proposition 3.20. Assume that $f$ is a convenient weighted homogeneous polynomial which is non-degenerate with respect to its Newton polyhedron. Denote by $v$ the primitive vector associated with $f$.

- $h(v)>0$ if and only if $e_{f}>0$, and more precisely $h(v)=m_{f}(v) e_{f}$.

- $h(v)=0$ if and only if $\operatorname{deg} \beta\left(\mathcal{A}_{k}(f)\right) \leq n-k$, with equality for infinitely many $k \in \mathbb{N}^{*}$.

- $h(v)<0$ if and only if $\operatorname{deg} \beta\left(\mathcal{A}_{k}(f)\right)<n-k$ for all $k \in \mathbb{N}^{*}$.

Remark 3.21. In [1], it is shown that the weights of a non-degenerate weighted homogeneous polynomial in two variable are invariants under blow-analytic equivalence, using the zeta function defined with the Euler characteristic of the homology of locally finite chains with closed supports [13]. Because of the good properties of the virtual Poincaré polynomial, we recover easily the analogous result, in the setting of blow-Nash equivalence. Indeed, the first exponent of the zeta function combined with the leading exponent give the weights. We mention moreover that, in the two variable case, a complete classification has been recently achieved by $\mathrm{S}$. Koike and A. Parusiński [14].

\section{Recovering the Weights}

We focus in this section on how to recover the weights of a convenient nondegenerate weighted homogeneous polynomials in three variables from its zeta function. In the two variable case, the result is immediate by Proposition 3.20, since $h(v) \geq 0$ as soon as the germ is singular.

Let $f\left(x_{1}, x_{2}, x_{3}\right) \in \mathbb{R}\left[x_{1}, x_{2}, x_{3}\right]$ be a weighted homogeneous polynomial whose Newton polyhedron is convenient. Denote by $w_{1}, w_{2}, w_{3} \in \mathbb{N}$ the weights and by $d \in \mathbb{N}$ the weighted degree of $f$. Recall that $v=\left(w_{1}, w_{2}, w_{3}\right)$ is the primitive generator of the 1-cone associated with $\Gamma_{+}(f)$, and that $m_{f}(v)=d$. Let $V_{1}=$ $\left(p_{1}, 0,0\right), V_{2}=\left(0, p_{2}, 0\right)$ and $V_{3}=\left(0,0, p_{3}\right)$ denote the vertexes of $\Gamma_{+}(f)$, where $p_{1}, p_{2}, p_{3} \in \mathbb{N}^{*}$ since $\Gamma_{+}(f)$ is convenient. Then $w_{i}=m_{f}(v) / p_{i}$ for $i=1,2,3$. Assume that $p_{1} \leq p_{2} \leq p_{3}$ without loss of generality.

Let $\gamma_{f}$ denote the compact 2-dimensional face of $\Gamma_{+}(f)$. Set $\gamma_{i}=\gamma_{f} \cap\left\{\nu_{i}=0\right\}$ for $i \in\{1,2,3\}$, so that the compact faces of the Newton polyhedron of $f$ are $\gamma_{f}$ 
in dimension two, $\gamma_{1}, \gamma_{2}, \gamma_{3}$ in dimension one and $V_{1}, V_{2}, V_{3}$ for the vertexes. Set $p_{i j}=\operatorname{lcm}\left(p_{i}, p_{j}\right)$ for $i \neq j \in\{1,2,3\}$ and recall that $d=m_{f}(v)=\operatorname{lcm}\left(p_{1}, p_{2}, p_{3}\right)$.

We are going to give a complete description of the $k$-th coefficient of the zeta function of $f$, for $k \in \mathbb{N}^{*}$. Theorem 2.6 describes these coefficients with respect to the contribution of each compact face $\gamma<\Gamma(f)$ of the Newton polyhedron of $f$, via the terms $P_{k}(\gamma)$ and $Q_{k}(\gamma)$ (understood as virtual Poincaré polynomials, cf. the introduction of section 3$)$ given by

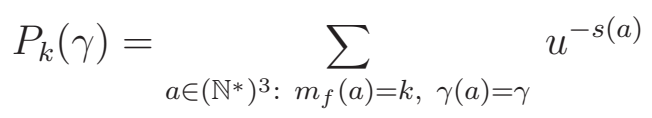

and

$$
Q_{k}(\gamma)=\sum_{a \in\left(\mathbb{N}^{*}\right)^{3}:} \sum_{m_{f}(a)<k, \gamma(a)=\gamma} u^{-k+m_{f}(a)-s(a)} .
$$

We begin with the terms of the form $P_{k}(\gamma)$.

Lemma 4.1. Let $k \in \mathbb{N}^{*}$ and $i \in\{1,2,3\}$. Set $\{j, s\}=\{1,2,3\} \backslash\{i\}$.

(1) The term $P_{k}\left(\gamma_{f}\right)$ is non-zero if and only if $d \mid k$, and in this case

$$
P_{k}\left(\gamma_{f}\right)=u^{-\left(\frac{k}{p_{1}}+\frac{k}{p_{2}}+\frac{k}{p_{3}}\right)} .
$$

(2) The term $P_{k}\left(\gamma_{i}\right)$ is non-zero if and only if $p_{j s} \mid k$, and in this case

$$
P_{k}\left(\gamma_{i}\right)=(u-1)^{-1} u^{-\left(\left\lfloor\frac{k}{p_{i}}\right\rfloor+\frac{k}{p_{j}}+\frac{k}{p_{s}}\right)} .
$$

(3) The term $P_{k}\left(V_{i}\right)$ is non-zero if and only if $p_{i} \mid k$, and in this case

$$
P_{k}\left(V_{i}\right)=(u-1)^{-2} u^{-\left(\frac{k}{p_{i}}+\left\lfloor\frac{k}{p_{j}}\right\rfloor+\left\lfloor\frac{k}{p_{s}}\right\rfloor\right)} .
$$

Proof. The face $\gamma<\Gamma(f)$ gives a non-zero contribution $P_{k}(\gamma)$ if and only if the set $\left\{a \in\left(\mathbb{N}^{*}\right)^{n}: m_{f}(a)=k, \gamma(a)=\gamma\right\}$ is not empty. Concerning $\gamma_{f}$, this set is equal to

$$
\left\{\lambda v: \lambda \in \mathbb{N}, m_{f}(\lambda v)=k\right\}= \begin{cases}\left\{\frac{k}{m_{f}(v)} v\right\} & \text { if } m_{f}(v)=d \mid k \\ \emptyset & \text { otherwise }\end{cases}
$$

and therefore if $d \mid k$ we have $P_{k}\left(\gamma_{f}\right)=u^{-s\left(\frac{k}{m_{f}(v)} v\right)}$, where $v=\left(\frac{m_{f}(v)}{p_{1}}, \frac{m_{f}(v)}{p_{2}}, \frac{m_{f}(v)}{p_{3}}\right)$. In the case of $\gamma_{i}$, this set is equal to

$$
\left\{\left(a_{i}, a_{j}, a_{s}\right) \in\left(\mathbb{N}^{*}\right)^{3}: a_{i} p_{i}>a_{j} p_{j}=a_{s} p_{s}=k\right\}= \begin{cases}\left\{\left(a_{i}, \frac{k}{p_{j}}, \frac{k}{p_{s}}\right): a_{i} \geq\left\lfloor\frac{k}{p_{i}}\right\rfloor+1\right\} & \text { if } p_{j s} \mid k, \\ \emptyset & \text { otherwise }\end{cases}
$$

and therefore

$$
P_{k}\left(\gamma_{i}\right)=\sum_{l \geq 0} u^{-\left(\left\lfloor\frac{k}{p_{i}}\right\rfloor+1+l+\frac{k}{p_{j}}+\frac{k}{p_{s}}\right)}=u^{-\left(\left\lfloor\frac{k}{p_{i}}\right\rfloor+1+\frac{k}{p_{j}}+\frac{k}{p_{s}}\right)} \frac{u}{u-1} .
$$

Finally, in the case of $V_{i}$, this set is equal to

$$
\begin{gathered}
\left\{\left(a_{i}, a_{j}, a_{s}\right) \in\left(\mathbb{N}^{*}\right)^{3}: a_{i} p_{i}=k, k<a_{j} p_{j}, k<a_{s} p_{s}\right\} \\
= \begin{cases}\left\{\left(\frac{k}{p_{i}}, a_{j}, a_{s}\right): a_{j} \geq\left\lfloor\frac{k}{p_{j}}\right\rfloor+1, a_{s} \geq\left\lfloor\frac{k}{p_{s}}\right\rfloor+1\right\} & \text { if } p_{i} \mid k, \\
\emptyset & \text { otherwise, }\end{cases}
\end{gathered}
$$


and therefore

$$
P_{k}\left(V_{i}\right)=\sum_{l_{1}, l_{2} \geq 0} u^{-\left(\frac{k}{p_{i}}+\left\lfloor\frac{k}{p_{j}}\right\rfloor+1+l_{1}+\left\lfloor\frac{k}{p_{s}}\right\rfloor+1+l_{2}\right)}=u^{-\left(\frac{k}{p_{i}}+\left\lfloor\frac{k}{p_{j}}\right\rfloor+1+\left\lfloor\frac{k}{p_{s}}\right\rfloor+1\right)}\left(\frac{u}{u-1}\right)^{2} .
$$

Now we focus on the terms of the form $Q_{k}(\gamma)$.

Lemma 4.2. For $k \in \mathbb{N}^{*}$ and $\gamma<\Gamma(f)$, we have

$$
Q_{k}(\gamma)=\sum_{1 \leq l<k} u^{-k+l} P_{l}(\gamma)
$$

Proof. By definition of $Q_{k}(\gamma)$ we have

$$
Q_{k}(\gamma)=\sum_{1 \leq l<k} \sum_{a \in\left(\mathbb{N}^{*}\right)^{3}:} u_{m_{f}(a)=l, \gamma(a)=\gamma} u^{-k+m_{f}(a)-s(a)}=\sum_{1 \leq l<k} u^{-k+l} P_{l}(\gamma) .
$$

Proposition 4.3. Let $k \in \mathbb{N}^{*}$. The coefficient of $t^{k}$ in the zeta function of $f$ is given by

$$
\beta\left(\mathcal{A}_{k}(f)\right)=\frac{(u-1) \xi_{k}}{u^{\left\lfloor\frac{k}{p_{1}}\right\rfloor+\left\lfloor\frac{k}{p_{2}}\right\rfloor+\left\lfloor\frac{k}{p_{3}}\right\rfloor}}+\sum_{1 \leq l<k} \frac{(u-1)^{2} \eta_{l}}{u^{k-l+\left\lfloor\frac{l}{p_{1}}\right\rfloor+\left\lfloor\frac{l}{p_{2}}\right\rfloor+\left\lfloor\frac{l}{p_{3}}\right\rfloor}}
$$

where

$$
\xi_{k}= \begin{cases}0 & \text { if } p_{i} \nmid k(i=1,2,3) \\ 1 & \text { if } p_{i} \mid k, p_{i j} \nmid k(i \neq j) \\ 1+u-\beta\left(\widehat{X}_{\gamma_{s}}\right) & \text { if } p_{i j} \mid k, d \nmid k,\{i, j, s\}=\{1,2,3\} \\ 1+u+u^{2}-\beta\left(\widehat{X}_{\gamma_{f}} \cup \widehat{X}_{\gamma_{1}} \cup \widehat{X}_{\gamma_{2}} \cup \widehat{X}_{\gamma_{3}}\right) & \text { if } d \mid k\end{cases}
$$

and

$$
\eta_{l}= \begin{cases}0 & \text { if } p_{i} \nmid l(i=1,2,3) \text { or } p_{i} \mid l, p_{i j} \nmid l(i \neq j) \\ \beta\left(\widehat{X}_{\gamma_{s}}\right) & \text { if } p_{i j} \mid l, d \nmid l,\{i, j, s\}=\{1,2,3\} \\ \beta\left(\widehat{X}_{\gamma_{f}} \cup \widehat{X}_{\gamma_{1}} \cup \widehat{X}_{\gamma_{2}} \cup \widehat{X}_{\gamma_{3}}\right) & \text { if } d \mid l .\end{cases}
$$

Proof. For a compact face $\gamma$ of $\Gamma_{+}(f)$, we know from Remark 2.1 that

$$
\beta\left(X_{\gamma}\right)=(u-1)^{3-\operatorname{dim} \gamma} \beta\left(\widehat{X}_{\gamma}\right) .
$$

Moreover, if $\gamma=V_{i}$ is a vertex, where $i=1,2,3$, then $\beta\left(X_{V_{i}}\right)=0$. Combined with the formula given in Theorem 2.6, we obtain that $\beta\left(\mathcal{A}_{k}(f)\right)$ is equal to the sum of the contribution of the compact facet $\gamma_{f}$

$$
(u-1)\left((u-1)^{2}-\beta\left(\widehat{X}_{\gamma_{f}}\right)\right) P_{k}\left(\gamma_{f}\right)+(u-1)^{2} \beta\left(\widehat{X}_{\gamma_{f}}\right) Q_{k}\left(\gamma_{f}\right),
$$

with the contribution of the 1-dimensional faces $\gamma_{1}, \gamma_{2}, \gamma_{3}$,

$$
(u-1)^{2} \sum_{i=1}^{3}\left((u-1)-\beta\left(\widehat{X}_{\gamma_{i}}\right)\right) P_{k}\left(\gamma_{i}\right)+(u-1)^{3} \sum_{i=1}^{3} \beta\left(\widehat{X}_{\gamma_{i}}\right) Q_{k}\left(\gamma_{i}\right),
$$


MOTIVIC INVARIANTS OF REAL POLYNOMIAL FUNCTIONS AND NEWTON POLYHEDRONS 23 and with the contribution of the vertexes $P_{1}, P_{2}, P_{3}$,

$$
(u-1)^{3} \sum_{i=1}^{3} P_{k}\left(V_{i}\right) \text {. }
$$

It remains to describe each contribution with respect to the relations between $p_{1}, p_{2}, p_{3}$ and $k$. We begin with $\xi_{k}$. By Lemma 4.1, it is clear that $\xi_{k}=0$ if none of the $p_{i}$ divides $k$ for $i=1,2$, 3. If $p_{i} \mid k$ but $p_{i j} \nmid k$ for $i \neq j$, then the only face contributing to $\xi_{k}$ is $V_{i}$ and by Lemma 4.1

$$
(u-1)^{3} P_{k}\left(V_{i}\right)=(u-1) u^{-\left(\frac{k}{p_{i}}+\left\lfloor\frac{k}{p_{j}}\right\rfloor+\left\lfloor\frac{k}{p_{s}}\right\rfloor\right)}
$$

so that $\xi_{k}=1$ as claimed. Assume that $p_{i j} \mid k$ but $d \nmid k$ and denote $\{i, j, s\}=$ $\{1,2,3\}$. Then the contribution to $\xi_{k}$ comes from the faces $V_{i}, V_{j}$ and $\gamma_{s}$, and this contribution is equal to

$$
\begin{gathered}
(u-1)^{2}\left((u-1)-\beta\left(\widehat{X}_{\gamma_{s}}\right)\right) P_{k}\left(\gamma_{s}\right)+(u-1)^{3} P_{k}\left(V_{i}\right)+(u-1)^{3} P_{k}\left(V_{j}\right) \\
=u^{-\left(\frac{k}{p_{i}}+\frac{k}{p_{j}}+\left\lfloor\frac{k}{p_{s}}\right\rfloor\right)}\left((u-1)\left((u-1)-\beta\left(\widehat{X}_{\gamma_{s}}\right)\right)+2(u-1)\right)
\end{gathered}
$$

by Lemma 4.1 , so that $\xi_{k}=1+u-\beta\left(\widehat{X}_{\gamma_{s}}\right)$ as expected. Finally, assume that $d \mid k$. Then all the faces contribute to $\xi_{k}$ and the contribution is equal to

$$
\begin{gathered}
(u-1) u^{-\left(\frac{k}{p_{i}}+\frac{k}{p_{j}}+\frac{k}{p_{s}}\right)}\left((u-1)^{2}-\beta\left(\widehat{X}_{\gamma_{f}}\right)+\sum_{i=1}^{3}\left((u-1)-\beta\left(\widehat{X}_{\gamma_{i}}\right)\right)+3\right) \\
=(u-1) u^{-\left(\frac{k}{p_{i}}+\frac{k}{p_{j}}+\frac{k}{p_{s}}\right)}\left(u^{2}+u+1-\beta\left(\widehat{X}_{\gamma_{f}}\right)-\sum_{i=1}^{3} \beta\left(\widehat{X}_{\gamma_{i}}\right)\right)
\end{gathered}
$$

so that $\xi_{k}=u^{2}+u+1-\beta\left(\widehat{X}_{\gamma_{f}} \cup \widehat{X}_{\gamma_{1}} \cup \widehat{X}_{\gamma_{2}} \cup \widehat{X}_{\gamma_{3}}\right)$ as expected.

Now we focus on $\eta_{l}$, with $1 \leq l<k$. First of all, the contribution of the terms of the form $Q_{k}(\gamma)$ to $\beta\left(\mathcal{A}_{k}(f)\right)$ is given by

$$
(u-1)^{2} \beta\left(\widehat{X}_{\gamma_{f}}\right) Q_{k}\left(\gamma_{f}\right)+(u-1)^{3} \sum_{i=1}^{3} \beta\left(\widehat{X}_{\gamma_{i}}\right) Q_{k}\left(\gamma_{i}\right),
$$

which is equal to

$$
(u-1)^{2} \beta\left(\widehat{X}_{\gamma_{f}}\right) \sum_{1 \leq l<k} u^{l-k} P_{l}\left(\gamma_{f}\right)+(u-1)^{3} \sum_{i=1}^{3} \beta\left(\widehat{X}_{\gamma_{i}}\right) \sum_{1 \leq l<k} u^{l-k} P_{l}\left(\gamma_{i}\right)
$$

by Lemma 4.2. In particular, using Lemma 4.1,

- if $p_{i} \nmid l$ for $i=1,2,3$, or if $p_{i} \mid l$ but $p_{i j} \nmid l$ for $j \neq i$, then $P_{l}\left(\gamma_{f}\right)=P_{l}\left(\gamma_{i}\right)=0$ for $1 \leq l<k$ and $i=1,2,3$, therefore $\eta_{l}=0$,

- if $p_{i j} \mid l$ with $i, j \in\{1,2,3\}$ but $d \nmid l$, then $\eta_{l}=\beta\left(\widehat{X}_{\gamma_{s}}\right)$, where $\{i, j, s\}=$ $\{1,2,3\}$,

- if $d \mid l$, then $\eta_{l}=\beta\left(\widehat{X}_{\gamma_{f}}\right)+\sum_{i=1}^{3} \beta\left(\widehat{X}_{\gamma_{i}}\right)$. 
Corollary 4.4. Let $k \in \mathbb{N}^{*}$. The degree of the virtual Poincaré polynomial of $\mathcal{A}_{k}(f)$ satisfies the bound

$$
\operatorname{deg} \beta\left(\mathcal{A}_{k}(f)\right) \leq 3-\left\lfloor\frac{k}{p_{1}}\right\rfloor-\left\lfloor\frac{k}{p_{2}}\right\rfloor-\left\lfloor\frac{k}{p_{3}}\right\rfloor .
$$

Remark 4.5. Note in particular that

- if $p_{1}<p_{2} \leq p_{3}$, then $\beta\left(\mathcal{A}_{p_{1}}(f)\right)=\frac{u-1}{u}$.

- if $p_{1}=p_{2}<p_{3}$, then $\beta\left(\mathcal{A}_{p_{1}}(f)\right)=\frac{(u-1)\left(1+u-\beta\left(\widehat{X}_{\gamma_{3}}\right)\right)}{u^{2}}$.

- if $p_{1}=p_{2}=p_{3}$, then $\beta\left(\mathcal{A}_{p_{1}}(f)\right)=\frac{(u-1)\left(1+u+u^{2}-\beta\left(\widehat{X}_{\gamma_{f}} \cup \widehat{X}_{\gamma_{1}} \cup \widehat{X}_{\gamma_{2}} \cup \widehat{X}_{\gamma_{3}}\right)\right)}{u^{3}}$.

However, in order to recover the weights of $f$, it will be enough to concentrate the study on some specific parts of the zeta function. Actually, it is enough to recover the integers $p_{1}, p_{2}$ and $p_{3}$, together with $m_{f}(v)$, from the zeta function of $f$ since $w_{i}=m_{f}(v) / p_{i}$ for $i=1,2,3$. Note that we already recover the multiplicity of $f$, that is $p_{1}$, as the order of the zeta function. Moreover we know from Proposition 3.20 how to recover the sign of $h(v)$.

In the particular case of $h(v)<0$ the function $f$ has only simple singularities in the sense of Arnold [2] and we already know how to recover the weights from [8].

In the general situation, namely when $h(v) \geq 0$, we obtain

$$
e_{f}=\frac{h(v)}{m_{f}(v)}=1-\frac{1}{p_{1}}-\frac{1}{p_{2}}-\frac{1}{p_{3}}
$$

by Proposition 3.11 and $m_{f}(v)$ by Corollary 3.14. Therefore it is sufficient to find $p_{2}$ or $p_{3}$ in order to recover all the weights. We are going to recover $p_{2}$ by a direct analysis of the first terms of the zeta function of $f$. More precisely, the idea is to recover $p_{2}$ in the zeta function as the first contribution that does not come from the vertex $\left(p_{1}, 0,0\right)$ of the Newton polyhedron of $f$. Recall that $A(f)$ denotes the set of Fukui invariants $A(f)=\left\{k \in \mathbb{N}^{*}: \mathcal{A}_{k} \neq \emptyset\right\}$.

We treat the cases $p_{1}$ even and $p_{1}$ odd separately. In the case $p_{1}$ is odd, note that $X_{\gamma_{3}}$ is not empty, so that $\beta\left(X_{\gamma_{3}}\right) \neq 0$ and therefore $A(f) \cap \mathbb{N}_{\geq p_{12}}=\mathbb{N}_{\geq p_{12}}$.

Set

$$
\begin{aligned}
& \alpha=\min \left\{l \in \mathbb{N}^{*}: A(f) \cap \mathbb{N}_{\geq l}=\mathbb{N}_{\geq l}\right\}, \\
& \beta=\min \left\{l \in \mathbb{N}^{*}: \beta\left(\mathcal{A}_{l}\right) \neq 0, p_{1} \nmid l\right\} .
\end{aligned}
$$

As $p_{1}$ is odd, note that $\alpha \leq p_{12}$.

Lemma 4.6. Assume that $p_{1}$ is odd.

- If $p_{1} \nmid \beta-1$, then $p_{2}=\beta$.

- If $p_{1} \mid \beta-1$ and $\beta-1<\alpha$, then $p_{2}=\beta$.

- If $p_{1} \mid \beta-1$ and $\beta-1=\alpha$, then either $p_{2}=\beta-1$ or $p_{2}=p_{3}=\beta$.

Proof. If $p_{1}$ divides $p_{2}$, then $\alpha=p_{2}$ and $\beta=\alpha+1$, so that $p_{1}$ divides $\beta-1$. As $\beta=p_{2}$ if $p_{1}$ does not divide $p_{2}$, we obtain the first claim.

Now, if $p_{1}$ divides $\beta-1$, then either $p_{1}$ divides $p_{2}=\beta-1$ and $\alpha=p_{2}$ or $p_{1}$ divides $p_{2}-1$ and $p_{2}=\beta$.

In particular, if $p_{1} \mid \beta-1$ and $\beta=\alpha+1$, we obtain two possibilities for the value of $p_{2}$. We show below that only one of these possibilities gives the correct value for the sum $\frac{1}{p_{1}}+\frac{1}{p_{2}}+\frac{1}{p_{3}}$, except in one particular case that we need to treat separately. 
Lemma 4.7. Assume that $p_{1} \mid \beta-1$ and $\beta=\alpha+1$. Assume moreover that the value of $\frac{1}{p_{1}}+\frac{1}{p_{2}}+\frac{1}{p_{3}}$ is given. Then we can decide whether $p_{2}=\alpha$ or $p_{2}=\beta$, except in the cases $\left(p_{1}, p_{2}, p_{3}\right)=(3,4,4)$ or $(3,3,6)$.

Proof. Assume that $\left(p_{2}, p_{3}\right)=(\beta, \beta)$ and $\left(p_{2}^{\prime}, p_{3}^{\prime}\right)=(\alpha, l)$ with $l \geq \alpha$ satisfying

$$
\frac{1}{p_{2}}+\frac{1}{p_{3}}=\frac{1}{p_{2}^{\prime}}+\frac{1}{p_{3}^{\prime}}
$$

Then $l=\frac{\alpha(\alpha+1)}{\alpha-1}$ should be an integer, and therefore $\alpha=3$ and $l=6$.

In the case $p_{1}$ is even, it may be possible that $\alpha=\infty$ (only if $\beta\left(X_{\gamma_{f}}\right)=0$ ), and even $\beta=\infty$ (only if $p_{1}$ divides $p_{2}$ and $p_{3}$ ). Therefore we need to take care also about the coefficients of the zeta function, as described in Proposition 4.3. Denote by $J$ a third root of unity different from 1 and set

$$
\delta=\min \left\{l \in \mathbb{N}^{*}:-1 \text { or } J \text { is a zero of } \beta\left(\mathcal{A}_{l}\right)\right\} .
$$

Lemma 4.8. Assume that $p_{1}$ is even. Then $p_{2}=\beta$ if $\alpha=\beta-1$ and $\beta\left(\mathcal{A}_{\alpha}\right)=$ $u^{-\frac{\alpha}{p_{1}}}(u-1)$. In the other cases, $p_{2}=\min \{\alpha, \beta, \delta\}$.

Proof. We distinguish cases according to the divisibility of $p_{2}$ by $p_{1}$, and according to equality between $p_{2}$ and $p_{3}$.

- If $p_{1}$ does not divide $p_{2}$ and $p_{2}<p_{3}$, then $p_{2}=\beta$. Moreover $\beta \leq \delta$ by Proposition 4.3. We claim that necessarily $\beta \leq \alpha$. If not, the only possible case is $\alpha=\beta-1$ and so $p_{1}$ divides $\beta-1=p_{2}-1$. Since $p_{2}+1$ should be an element of $A(f) \cap \mathbb{N}_{\geq \alpha}$, then $p_{1}$ divides $p_{2}+1$ also, and so $p_{1}=2$. Similarly $p_{2}+2$ should be an element of $A(f) \cap \mathbb{N}_{\geq \alpha}$, and so $p_{3}=p_{2}+2$ and thus $\left(p_{1}, p_{2}, p_{3}\right)$ has the form $\left(2, p_{2}, p_{2}+2\right)$ with $p_{2}$ odd. Finally $p_{2}+4$ should be an element of $A(f) \cap \mathbb{N}_{\geq \alpha}$, so $p_{2}+4 \geq \operatorname{lcm}\left(2, p_{2}\right)=2 p_{2}$. As a consequence $\left(p_{1}, p_{2}, p_{3}\right)=(2,3,5)$ and thus $h(v)<0$, which has been excluded in our discussion.

- If $p_{1}$ does not divide $p_{2}$ and $p_{2}=p_{3}$, then $p_{2}=\beta$ and $\beta \leq \delta$ by Proposition 4.3. Moreover $\beta \leq \alpha$ unless $p_{1}$ divides $p_{2}-1$ and $\beta\left(X_{\gamma_{1}}\right) \neq 0$. In that case, $\beta=\alpha+1 \leq \delta$ and moreover $\beta\left(\mathcal{A}_{\alpha}\right)=u^{-\frac{\alpha}{p_{1}}}(u-1)$ by Proposition 4.3. This case gives the exceptional case in the statement of the lemma.

- If $p_{1}$ divides $p_{2}$, assume first that $p_{2}<p_{3}$. Then $p_{2}=\delta$ if $\beta\left(X_{\gamma_{3}}\right)=0$, and then $\delta \leq \min \{\alpha, \beta\}$. If however $\beta\left(X_{\gamma_{3}}\right) \neq 0$, then $p_{2}=\alpha \leq \min \{\beta, \delta\}$.

In the latter case, note that $\beta\left(\mathcal{A}_{\alpha}\right)=u^{-\frac{\alpha}{p_{1}}-1}\left(u^{2}-1-(u-1) \beta\left(\widehat{X}_{\gamma_{3}}\right)\right)$ with $\beta\left(\widehat{X}_{\gamma_{3}}\right)$ even (indeed $\beta\left(\widehat{X}_{\gamma_{3}}\right)$ is equal to the number of real solutions of a real polynomial of even degree not vanishing at zero, with only simple real roots because $f$ has isolated singularities).

- If $p_{1}$ divides $p_{2}=p_{3}$, we have to distinguish the cases where $\alpha$ is infinite or not. If $\alpha \neq \infty$ then $p_{2}=\alpha \leq\{\beta, \delta\}$ since $\left[X_{\gamma_{f}}\right] \neq 0$. Finally if $p_{1}$ divides $p_{2}=p_{3}$ and $\alpha=\infty$, then $p_{2}<\beta$ and $J$ is a zero of $\beta\left(\mathcal{A}_{p_{2}}\right)$ by Proposition 4.3 , therefore $p_{2}=\delta$.

Theorem 4.9. Convenient weighted homogeneous polynomials which share the same zeta functions have the same weights. 
Proof. If $h(v)<0$ we refer to [8]. Otherwise, the discussion in section 4 shows how to recover $p_{1}, p_{2}$ and $\frac{1}{p_{1}}+\frac{1}{p_{2}}+\frac{1}{p_{3}}$ except in the particular case where $p_{1}=3$ and $\frac{1}{p_{1}}+\frac{1}{p_{2}}+\frac{1}{p_{3}}=\frac{5}{6}$. Therefore it suffices to distinguish the cases $\left(p_{1}, p_{2}, p_{3}\right)=(3,4,4)$ and $(3,3,6)$.

A direct computation shows that $\beta\left(\mathcal{A}_{4}\right)=u^{-3}(u-1)^{2}$ if $\left(p_{1}, p_{2}, p_{3}\right)=(3,3,6)$ whereas $\beta\left(\mathcal{A}_{4}\right)=u^{-3}\left(u^{2}-1-(u-1) \beta\left(\widehat{X}_{\gamma_{1}}\right)\right)$ if $\left(p_{1}, p_{2}, p_{3}\right)=(3,4,4)$, so that the spaces of arcs of level 4 are different, except when $\beta\left(\widehat{X}_{\gamma_{1}}\right)=2$. However $\beta\left(\mathcal{A}_{5}\right)=$ $u^{-4}(u-1)^{2}$ if $\left(p_{1}, p_{2}, p_{3}\right)=(3,3,6)$ whereas $\beta\left(\mathcal{A}_{5}\right)=u^{-4}(u-1) \beta\left(\widehat{X}_{\gamma_{1}}\right)$ if $\left(p_{1}, p_{2}, p_{3}\right)=$ $(3,4,4)$, so at level 5 the spaces of arcs are different.

\section{REFERENCES}

[1] O. M. Abderrahmane, Weighted homogeneous polynomials and blow-analytic equivalence, Singularity theory and its applications, 333-345, Adv. Stud. Pure Math., 43, Math. Soc. Japan, Tokyo, (2006)

[2] V. Arnold, S. Goussein-Zadé, A. Varchenko, Singularity of differentiable maps, Birkhauser, Boston (1985)

[3] J. Denef, K. Hoornaert, Newton polyhedra and Igusa's local zeta function, J. Number Theory 89 (2001), no. 1, 31-64

[4] J. Denef, F. Loeser, Caractéristiques d'Euler-Poincaré, fonctions zêta locales et modifications analytiques, J. Amer. Math. Soc. 5 (1995), 705-720

[5] J. Denef, F. Loeser, Germs of arcs on singular algebraic varieties and motivic integration, Invent. Math. 135 (1999), no. 1, 201-232

[6] J. Denef, F. Loeser, Geometry on arc spaces of algebraic varieties, Proceedings of 3rd European Congress of Mathematics, Barcelona, Progress in Mathematics 201, 327-348 (2001), Birkhuser

[7] G. Fichou, Motivic invariants of Arc-Symmetric sets and Blow-Nash Equivalence, Compositio Math. 141 (2005) 655-688

[8] G. Fichou, Blow-Nash type of simple singularities, J. Math. Soc. Japan, 60 no. 2 (2008), $445-470$

[9] T. Fukui, Seeking invariants for blow-analytic equivalence, Compositio Math. 105 (1997), no. 1, 95-108

[10] T. Fukui, S. Koike, T.-C. Kuo, Blow-analytic equisingularities, properties, problems and progress, Real Analytic and Algebraic Singularities (T. Fukuda, T. Fukui, S. Izumiya and S. Koike, ed), Pitman Research Notes in Mathematics Series, 381 (1998), pp. 8-29

[11] T. Fukui, L. Paunescu, On blow-analytic equivalence, Arc spaces and additive invariants in real algebraic geometry, Panoramas et Synthèses, SMF 26 (2008) 87-125

[12] G. Guibert, Espaces d'arcs et invariants d'Alexander, Comment. Math. Helv. 77 (2002), no. 4, $783-820$

[13] S. Koike, A. Parusiński, Motivic-type invariants of blow-analytic equivalence, Ann. Inst. Fourier (Grenoble) 53 (2003), no. 7, 2061-2104

[14] S. Koike, A. Parusiński, Blow-analytic equivalence of two variable real analytic function germs, J. Algebraic Geom. 19 (2010), no. 3, 439-472

[15] T.-C. Kuo, On classification of real singularities, Invent. Math. 82 (1985), 257-262

[16] C. McCrory, A. Parusiński, Virtual Betti numbers of real algebraic varieties, C. R. Math. Acad. Sci. Paris 336 (2003), no. 9, 763-768

[17] T. Nishimura, Topological invariance of weights for weighted homogeneous singularities, Kodai Math. J. 9 (1986), no. 2, 188-190

[18] O. Saeki, Topological Invariance of Weights for Weighted Homogeneous Isolated Singularities in $\mathbb{C}^{3}$, Proceedings of the American Mathematical Society, Vol. 103, No. 3 (Jul., 1988), 905-909

[19] K. Saito, Quasihomogene isolierte Singularitaten von Hyperflachen, Invent. Math. 14 (1971), 123-142 
MOTIVIC INVARIANTS OF REAL POLYNOMIAL FUNCTIONS AND NEWTON POLYHEDRONS 27

[20] E. Yoshinaga, M. Suzuki, Topological types of quasihomogeneous singularities in $\mathbb{C}^{2}$, Topology 18 (1979), no. 2, 113-116

IRMAR (UMR 6625), Université de Rennes 1, Campus de Beaulieu, 35042 Rennes Cedex, France

Department of Mathematics, Faculty of Science, Saitama University, Saitama, 338-8570, JAPAN 